Elsevier Editorial System(tm) for Placenta Manuscript Draft

Manuscript Number: PLAC-S-20-00253R2

Title: Ultrasound-histopathologic features of the utero-placental interface in placenta accreta spectrum

Article Type: Original article

Keywords: Placenta accreta; invasive placenta; placenta increta; uterine scar; placental basal plate; uterine vasculature

Corresponding Author: Professor Eric Jauniaux, MD, PhD, FRCOG

Corresponding Author's Institution: Royal Free and University College London

First Author: Eric Jauniaux, MD, PhD, FRCOG

Order of Authors: Eric Jauniaux, MD, PhD, FRCOG; Nurit Zosmer; Devi Subramanian; Hizbullah Shaikh; Graham J Burton

Abstract: Introduction: The objective of this study was to evaluate the relationship between utero-placental vascular changes on ultrasound imaging and histopathologic findings according to the grade of villous invasion in placenta accreta spectrum (PAS).

Methods: The ultrasound features of 31 patients with singleton pregnancies diagnosed prenatally with low-lying/placenta previa accreta were compared with histopathology findings following caesarean hysterectomy $(n=25)$ or partial myometrial resection $(n=6)$. The number and degree of transformation of arteries within the superficial layer of myometrium were recorded. Cytokeratin 7 (CK7) immunohistochemistry was used to complement $\mathrm{H} \& \mathrm{E}$ analysis.

Results: All 31 patients presented with loss of clear zone, myometrial thinning and placenta lacunae. Subplacental hypervascularity and lacunae feeder vessels were found in 25 and nine cases, respectively. Large recent intervillous thromboses were found in one case with adherent villi and 12 cases with invasive villi, and showed a significantly different distribution according to lacunae scores. Thick basal plate fibrinoid deposits in all the areas of abnormally adherent and invasive villous tissue There was no significant difference in the mean count of partially remodeled vessels or vessels completely lacking remodeling according to the lacunae score and grade of placental invasiveness. EVT cells were arranged in superficial confluent sheets or superficial irregular clusters, or were scattered deep below the basal plate.

Conclusion: Placental ultrasound and histopathologic features associated with PAS are more pronounced in invasive cases suggesting that they are secondary to the haemodynamic effects of abnormally deep placentation and transformation of the radial and arcuate arteries. 


\section{PLACENTA Manuscript Submission PLAC-S-20-00253 Ultrasound-histopathologic features of the utero-placental interface in placenta accreta spectrum}

\section{Answers to reviewer comments:}

Reviewer \#1: This is a descriptive study of 31 pregnancies complicated by either adherent or invasive placenta.

The authors compare the ultrasound features prior to delivery with analysis of the uteroplacental histology after delivery.

Their conclusions are that more placental lacunae, intervillous thrombi (IVT) and subplacental vascularity are more common in placentas with invasion.

They also examined the transformation of maternal arterioles in the myometrium but did not find any significant correlations in this regard.

Overall, the manuscript is well-presented, and the studies well done.

We thank the reviewer for his/her constructive comments

A few comments follow:

1) This is a descriptive study and perhaps part of the reason no real changes in maternal vessel remodeling were seen because these cases all have creta of one form or another. A true control group is not used in this study. A paragraph on the limitations of the study could highlight this in the discussion OR addition of a control group from hysterectomies without PAS would strengthen the study.

We agree that the only true control group would be caesarean hysterectomies for placenta previa non-accreta but those procedures are not performed anymore in high income countries as primary caesarean hysterectomies have been replaced by conservative management. However, they were more often performed in the 1950' and 1960' (when the prevalence of C-section was 6-8\%) and we have regularly used the Boyd and Hamilton collection at the CTR for our work on placentation including for a recent publication on the pathophysiology of PAS (ref 23). We have added a paragraph in the discussion to highlight this important point.

2) This reader was sometimes confused about the use of "invasive" and whether this was strictly a histology term or sometimes used as a radiology term. Clarification in Table 2 would help. Adherent and invasive, as defined by histologic examination I assume at the top of the columns are histology terms. Along those lines, the methods never really describe the histologic grades of PAS (adherent and invasive, I assume) and how they are defined. See line 140. I suggest adding some detail about the grading system used.

Changed as suggested.

3) The conclusion in the abstract is too strongly worded "are a consequence

of...." This study doesn't prove cause and effect, only associations. I suggest rewording the conclusion in the abstract.

Changed as suggested. 
4) The introduction is a little long and a little too far off topic. Some of it seemed not relevant to the study at hand (ie discussing the prevalence of PAS in primigravids).

Changed as suggested.

5) I was unsure how the histology explained or correlated the "subplacental hypervascularity". I assume this is not IVT, but rather the vascularity of the myometrium. Did arterial vessel counts differ in the myometrium amongst the cases? I am not sure this data was presented? Were there more venous channels? Other explanations? I wonder how vessel counts in the myometrium would compare with controls, if they were included.

This an ultrasound imaging description which corresponds to the increase in vascularity of the placental bed area. As in most cases, this area is also distorted by scar tissue and there is very little myometrium left it is difficult to be more specific from an anatomical point of view and it will be easier for clinicians if we stick to the ultrasound terminology. We have added a sentence in the discussion to "bridge the gap". We did not count the number of venous channels but in the hysterectomy specimens of the Boyd and Hamilton collection, there is no transformation of the radial or arcuate arteries.

6) I suggest a final paragraph in the discussion on the strengths and limitations of this study and summarizing the main findings one more time.

Added as suggested including the point raised in comment 1)

Reviewer \#2: The aim of this study is "to evaluate the relationship between uteroplacental vascular changes on ultrasound imaging and histopathologic findings according to the grade of villous invasiveness". The authors claim to demonstrate "a direct relationship between the vascular changes observed on ultrasound imaging in PAS and histopathologic findings" (meaning depth/grade of invasion).

Increased uterine vascularity is a hallmark of PAS, and the major cause of hemorrhagic morbidity, so understanding its pathophysiology is an important area of research.

However, it is difficult to interpret their findings without more description of their methods and more clinical information about their cohort. Also, the specific research hypothesis being tested needs to be stated more clearly.

The clinical characteristics was described in detailed at the beginning of the results (3.1 line 184-193). The study aim was clarified as suggested at the end of the Introduction.

1. The greatest source of my confusion was the lack of a specific definition of "invasion" or "villous invasiveness" or "invasive villi". Invasion seems to be defined in different ways throughout the paper for both pathology and ultrasound. This problem is compounded by the non-uniform terminology used for the pathology diagnosis of PAS (accreta/increta in some places, invasive/adherent/non-accreta in others, grade in others).

Examples: 
"Our study group included 31 patients with singleton pregnancies diagnosed prenatally with low-lying/placenta previa accreta." - They use of the term accreta, implying no-invasion, yet a subset of cases did show "invasion".

All terms defining invasion were replaced with invasive villi or villous invasion to avoid any confusion.

"Cases confirmed to be non-accreta placenta previa were excluded from the study." If the index diagnosis for their database search was an ultrasound diagnosis of lowlying/placenta previa accreta, then on what basis did they exclude cases? How did an ultrasound diagnosis of accreta get converted to "non-accreta placenta previa"? If these were false positive diagnoses based on ultrasound, overturned by pathology, wouldn't these women be the controls for the current study (relating ultrasound findings to histology)?

As all cases included in this study were previa + PAS confirmed by histopathology we did not include cases that were simply previa on ultrasound and which did therefore not require a hysterectomy and we have removed this sentence from the M\&M to avoid any confusion.

"All slides were examined by a specialist perinatal pathologist, and the histology report documented the presence and grade of villous invasion. The presence or absence of decidua was assessed by cell morphology." - What histologic features were used to determine the grade of invasion? Is the "absence of decidua" a separate variable or is it part of that grade?

As shown by Luke et al (ref 3 ) all three grades of PAS, creta, increta and percreta may co-exist in the same specimen. The absence of decidua can be a misleading sign of adherent PAS as the decidua becomes thinner and discontinuous with advancing gestation. A sentence was added to the M\&M to define the different grades of PAS.

"Cytokeratin 7 (CK7) immunohistochemistry was used to complement H\&E analysis, by highlighting EVT cells in 55 samples with evidence of villous invasion" - Does this imply that invasion is determined by the pattern of EVT infiltration

This happens simultaneously and is due to the scar damage to the myometrium allowing abnormal changes to the deep myometrial tissue and circulation as oppose to superficial myometrium and spiral arteries as shown in numerous prior studies on normal placentation (see paragraph in the introduction lines $\mathbf{7 0 - 7 8}$ which was amended slightly to clarify this point).

Line 224 comes close to a definition: "The invasive areas were characterized by variable and irregular villous ingrowth into the myometrium resulting in irregular, undulating basal plates with thick fibrin deposition surrounding all invasive villi. Creta areas showed chorionic villi in direct contact with the myometrium without interposing decidua basalis (Fig 2B) and were found in both adherent and invasive cases (Fig 2B). In the latter, these areas were adjacent to the foci of invasive villi." - Does this imply that invasion is diagnosed based on the shape of the placenta/myometrial interface and location/depth in the myometrium relative to adjacent decidua? That seems like a great definition if applied uniformly. We agree and it was not clearly defined by previous histopathology studies. We hope it will help pathologists to more accurately diagnose the different grades of PAS. 
2. Introduction: The introduction does not provide a context for the study design; there is no clear hypothesis being tested. The paper is about ultrasound, but the introduction is just a long review of the pathophysiology of PAS with very little literature background on diagnostic ultrasound (eg. sensitivity/specificity of specific ultrasound features of PAS, variation of these features with gestational age and clinical presentation).

Added as suggested

\section{Methods:}

A. Ultrasound examinations:

Did these women get serial ultrasound; were the findings consistent between scans (as a quality check, and as a measure of disease progression), were some features more common at earlier/later gestational age, and was correlation with pathology better/worse at early/later gestational age? Some of this is touched on (lines 203$210)$, but it is not clearly presented. The time range between last scan and delivery is not given.

Added as suggested (serial ultrasound examinations from 11-14 weeks were only available in 14 cases and these data were not included in this study as we focussed on the ultrasound findings obtained at the last scan before delivery)

\section{B. Cohort:}

The population had a large fraction of premature deliveries (29 to 37, mean: 35.3 weeks); was this a population unusual for published PAS cohorts?

There should be a section of the paper on discrepancies between ultrasound and pathology, perhaps with a control group of women with false positive ultrasound diagnosis of PAS. Some of these women may have had hysterectomies (for pathology controls), others would serve as controls for the clinical/ultrasound features (including placental location/size/myometrial thickness/bleeding at delivery). We are currently working on a prospective study to address some of these issues but in our experience (and that of team with similar expertise in prenatal ultrasound) the rate of false positive and false negative is less than $5 \%$. The data of the present study combined with those we published recently on how to obtain quality macroscopic examination (ref 34) have been essential in developing a new protocol. Quality and detailed histopathologic confirmation of the prenatal diagnosis have been the main issue of the vast majority of previously published cohort studies.

C. Histology: "villi invading vessels wall"(not illustrated) and "villous emboli" are not standard terms. Although prominent in PAS, villi can occasionally be seen in uterine veins during normal pregnancy; what are they describing?

This point was clarified in the discussion. We only identified villi crossing into the wall of a utero-placental artery in one case, suggesting that this may be an anecdotal finding (image below). This image can be added to the manuscript if necessary.

Clusters of villi were found in the lumen of intramyometrial vessels in 8 invasive cases. Similar findings were reported by C. Parra-Herran, B. Djordjevic (ref 28) and it is not something that we have noted in hysterectomy 
specimens from the CTR collection.

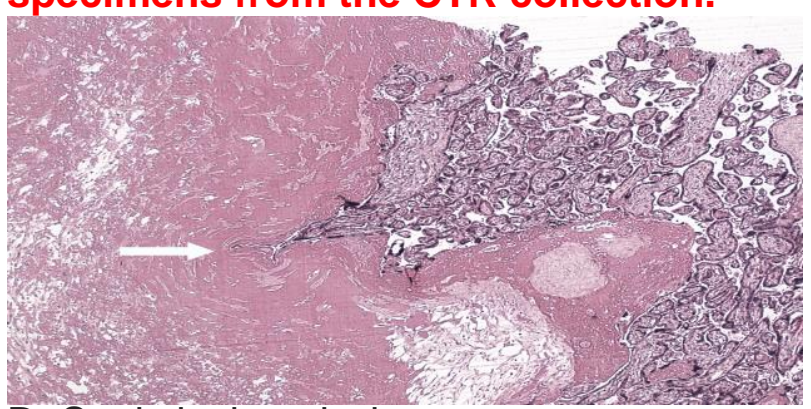

D. Statistical analysis.

It's unclear how they are analyzing their data, and to what end? What is the hypothesis being tested?

The main analysis is presented in Tables 1 and 2. "The data were separated into subgroups according to placental lacunae score and grade of placental adherence (creta) versus invasiveness (increta and percreta)." - Why was this approach chosen?

This point was clarified in both the introduction and M\&M following comments from both reviewers.

\section{Results:}

A. Again, the criteria for invasion are unclear.

For example, in table 2, I don't understand how 7 of 9 cases without invasion ("Adherent") have myometrial thickness $<1 \mathrm{~mm}$ on ultrasound? And, I don't accept their statement at face value:

"myometrial thickness on ultrasound ... decreases rapidly after 32 weeks when the lower uterine segment is further stretched by the combined action of the fetal presentation and Braxton-Hicks uterine contractions [23]." This statement may be true, but I would not guess that physiologic change would result in myometrium < $1 \mathrm{~mm}$. If so, I would expect a very high rate of over-diagnosis (of percreta) in cases of low-lying placentas. At minimum, they need a reference other than their own review article. Was there an attempt to correlate myometrial thickness, vascularity and lacunar score to clinical outcome (such as blood loss during surgery or bladder injury)? This might help explain the discrepancies between ultrasound and pathology diagnosis.

Ultrasound measurements of myometrial thickness have not been standardised and in particular not be adjusted for gestational age and parity in cases of PAS. We agree that this can be subjective as we find a thin myometrial thickness $(<2 \mathrm{~mm})$ in the third trimester of all cases of previa with or without PAS with prior C-section(s). Myometrial thickness has been evaluated extensively in the context of the risk of uterine rupture in VBAC/ToS and we have added 2 references ( $32 \& 33$ ) to support this statement.

B. In Figure 1, panel C, the caption describes the placenta, but the placenta is not shown in the image. Panel $D$ is not described in the legend, and is not labeled. What features indicate that this is invasive/increta?

With respect, this comment is a bit unfair. Panel $\mathrm{C}$ shows the hysterectomy specimens with the $\mathrm{C}$-section incision and the caption does not say that the placenta is visible. We have changed that caption to clarify this point. Panel D 
is described and labelled. This is quite obviously a typo with D missing.

5. Discussion:

Their major finding is "a direct relationship between the vascular changes observed on ultrasound imaging in PAS and histopathologic findings". Therefore, the focus of the discussion should be on the anatomic and physiologic basis for hypervascularity, and how it's linked to invasion, and the reliability of ultrasound to distinguish hypervascularity (more and abnormal vessels) from increased blood flow through normal, but dilated vessels. Why are they writing so extensively about decidual thickness, reduced spiral arteriole modeling, and perivillous fibrin?

This is also an unfair comment as we are the discussing the main findings of this study, which are that the vascular changes associated with PAS are due to the abnormal transformation of the deep uterine arteries which leads to the hypervascularity of the utero-placental interface and development of lacunae seen on ultrasound and the secondary development of vascular lesions such as intervillous thrombosis and fibrin deposition.

They do mention a pertinent negative finding of the study:

"the myometrial thickness was found to be less than $<1 \mathrm{~mm}$ in 24 out of 31 cases on ultrasound examination (Table 1) and it was not related to the grade of villous invasiveness (Table 2)."

- This seems to imply that vascularity and lacunar score are more reliable than ultrasound estimate of myometrial thickness. This is a secondary conclusion of the paper that needs discussion in the context of published ultrasound studies.

These points were clarified in the discussion. 


\section{Please wait...}

If this message is not eventually replaced by the proper contents of the document, your PDF viewer may not be able to display this type of document.

You can upgrade to the latest version of Adobe Reader for Windows ${ }$, Mac, or Linux ${ }^{\circledR}$ by visiting http://www.adobe.com/go/reader_download.

For more assistance with Adobe Reader visit http://www.adobe.com/go/acrreader.

Windows is either a registered trademark or a trademark of Microsoft Corporation in the United States and/or other countries. Mac is a trademark of Apple Inc., registered in the United States and other countries. Linux is the registered trademark of Linus Torvalds in the U.S. and other countries. 


\section{Ultrasound-histopathologic features of the utero-placental interface in placenta accreta spectrum}

\section{Abstract}

Introduction: The objective of this study was to evaluate the relationship between utero-placental vascular changes on ultrasound imaging and histopathologic findings according to the grade of villous invasion in placenta accreta spectrum (PAS). Methods: The ultrasound features of 31 patients with singleton pregnancies diagnosed prenatally with low-lying/placenta previa accreta were compared with histopathology findings following caesarean hysterectomy $(n=25)$ or partial myometrial resection $(n=6)$. The number and degree of transformation of arteries within the superficial layer of myometrium were recorded. Cytokeratin 7 (CK7) immunohistochemistry was used to complement H\&E analysis.

Results: All 31 patients presented with loss of clear zone, myometrial thinning and placenta lacunae. Subplacental hypervascularity and lacunae feeder vessels were found in 25 and nine cases, respectively. Large recent intervillous thromboses were found in one case with adherent villi and 12 cases with invasive villi, and showed a significantly different distribution according to lacunae scores. Thick basal plate fibrinoid deposits in all the areas of abnormally adherent and invasive villous tissue There was no significant difference in the mean count of partially remodeled vessels or vessels completely lacking remodeling according to the lacunae score and grade of placental invasiveness. EVT cells were arranged in superficial confluent sheets or superficial irregular clusters, or were scattered deep below the basal plate. 
Conclusion: Placental ultrasound and histopathologic features associated with PAS are more pronounced in invasive cases suggesting that they are secondary to the haemodynamic effects of abnormally deep placentation and transformation of the radial and arcuate arteries. 


\section{HIGHLIGHTS}

Lacunae in invasive placentation are due to high velocity flows entering the intervillous space.

- Lacunae and recent intervillous thromboses are associated with invasive placentation due to high velocity flow from deep large uterine arteries.

- Large recent intervillous thromboses lesions are common in invasive placentation.

- Invasive villi samples are surrounded by thick fibrin depositions and villi are found in uterine vessels due to abnormal flow pattern.

- These effects are not associated with the extent of trophoblastic vascular remodelling and may be influenced by the amount of scar tissue in the sample.

- Scar tissue may impact on myometrial vascular remodeling and extravillous trophoblast migration.
Formatted: Font: (Default) Arial

Formatted: Normal, Indent: Left: $-0.63 \mathrm{~cm}$

Formatted: Normal

Formatted: Font: (Default) Arial, Fon color: Custom Color(RGB $(34,34,34))$

Formatted: Font: (Default) Arial

Formatted: Font: (Default) Arial, Fon color: Custom Color(RGB $(34,34,34))$

Formatted: Font: (Default) Arial, Fo color: Custom Color(RGB(34,34,34))

Formatted: Font: (Default) Arial

Formatted: Font: (Default) Arial, Fon color: Custom Color(RGB $(34,34,34))$ Pattern: Clear (White)

Formatted: Font: Font color: Auto, English (United Kingdom)

Formatted: Indent: Left: $1.27 \mathrm{~cm}$, No bullets or numbering

Formatted: Font: Font color: Auto, English (United Kingdom)

Formatted: Font: (Default) Arial

Formatted: Normal, No bullets or numbering 


\section{1}

2

\title{
Ultrasound-histopathologic features of the utero-placental interface in placenta accreta spectrum
}

\author{
Eric Jauniaux ${ }^{a}$, Nurit Zosmer ${ }^{\text {b }}$, Devi Subramanian ${ }^{b}$, Hizbullah Shaikh ${ }^{c}$, Graham J \\ Burton $^{\text {d }}$ \\ ${ }^{\text {a} E G A ~ I n s t i t u t e ~ f o r ~ W o m e n ' s ~ H e a l t h, ~ F a c u l t y ~ o f ~ P o p u l a t i o n ~ H e a l t h ~ S c i e n c e s, ~}$ \\ University College London (UCL), London, UK. \\ 'The Fetal Medicine Research Institute, Harris Birthright Research Centre, King's \\ College Hospital, London, UK \\ 'Department of Histopathology (Dr Shaikh), King's College Hospital, London, UK \\ dThe Centre for Trophoblast Research (CTR), Department of Physiology, \\ Development and Neuroscience, University of Cambridge, Cambridge, United \\ Kingdom.
}

Conflict of interest: none

Word count main text: 3740

No funding was obtained for this study.

Corresponding author: Professor Eric Jauniaux, Institute for Women's Health, University College London, 86-96 Chenies Mews, London WC1E 6HX, UK.

Telephone numbers: $+44 / 207 / 3908113$

Fax: +44/207/3908115

E-mail: e.jauniaux@ucl.ac.uk 


\section{Introduction}

Placenta accreta spectrum (PAS) is a modern disorder of human placentation which is graded according to the degree of villous adherence to, or invasion into, the uterine wall by the placental tissue [1]. Until the end of the last century, PAS was a very rare complication of pregnancy, affecting $<1$ in 10,000 livebirths [2,3]. Following the exponential increase in the number of caesarean deliveries during the last two decades, the prevalence of PAS has risen to $<1$ in 1,000 livebirths [4] with some authors reporting prevalence as high as $1 \%$ of livebirths in their population $[5,6]$. Epidemiological data on prevalence of PAS are highly heterogenous due to the lack of a standardised terminology [7] and confirmation of the degree of villous invasion at birth in most case series $[8,9]$.

Any form of damage, even small, to the integrity of the endometrial uterine lining following a uterine curettage, myomectomy, or endometrial resection has been associated with the development of both adherent and invasive accreta placentation in subsequent pregnancies [10-12]. However, in the majority of these cases there was no evidence of myometrial invasion. Similarly, in the first detailed case series of accreta placentation reported in 1937 by Irving and Hertig [2] all 18 cases were described as abnormally adherent with no macroscopic nor histological evidence of myometrial villous invasion. By contrast, the current trend of multiple caesarean deliveries often leads to the development of large and deep myometrial defects, and there is a direct association between blastocyst implantation in a caesarean scar and the development of placenta previa accreta $[13,14]$. More than $90 \%$ of cases of PAS are now found in women with a history of prior caesarean delivery presenting with a low-lying or placenta 
previa [15], suggesting a tropism of the blastocyst for the scar area [16]. In contrast to uterine epithelial layers that heal by regeneration and recolonization of scar areas, the myometrium, does not heal by regenerating muscle fibers and myometrial scar tissue often presents with myofibre disarray, tissue edema, inflammation and elastosis [17]. These data support the concept that in PAS the trophoblast is biologically normal but becomes excessively invasive secondary to implantation into an anatomically abnormal uterine bed resulting from a surgical scar $[10,16]$.

Placentation in the human in physiologically invasive [18]. In normal pregnancies, the interstitial extravillous trophoblast (EVT) invade the uterine wall as far as the inner third of the myometrium [19], also called the junctional-zone (JZ), where they fuse to form multinucleated trophoblast giant cells (MNGCs) [20]. In the weeks following implantation, EVT cells are found both within and around the spiral arteries in the central area of the placental bed. Both endovascular and interstitial EVT invasion are associated with the physiological conversion of the terminal part of the uterine circulation down to the level of the spiral arteries in the $\mathrm{JZ}$ of the inner third of the myometrium. In PAS the EVT cells are increased in size and number, and the depth of their myometrial invasion is greater [21]. There is also a failure of fusion of the EVT to form MNGCs. As a result, the EVT come into contact with, and invade into the walls of, the radial and even the arcuate arteries.

Tabsh et al. [22] in 1982 were the first to report on the prenatal diagnosis of a case of placenta increta using grey-scale ultrasound imaging. The use of colour-Doppler imaging (CDI) two decades later has enabled a better visualisation of the vascular changes associated with PAS before birth [7]. Increased sub-placental hypervascularity 
and the presence of intra-placental lacunae are the most prominent features of invasive PAS prenatally on ultrasound [23]. Only a few authors have recently reported on the histopathology of the placenta and utero-placental interface in PAS [24-29], but none have studied the possible link between prenatal ultrasound features and placental pathologic findings. Similarly, authors of ultrasound imaging cohort studies have rarely provided detailed histopathologic findings beyond the classical description of absent decidua or the presence of invasive villi on histologic examination [7-9]. The purpose of our study was twofold: first to evaluate the impact of the changes in the utero-placental and intervillous circulations seen on ultrasound imaging on histopathologic features in PAS, and secondly to compare the extensiveness of EVT migration and uterine arterial transformation according to the grade of villous invasion.

\section{Material and Methods}

Our study group included 31 patients with singleton pregnancies diagnosed prenatally with low-lying/placenta previa accreta and confirmed by histopathology. All patients delivered at King's College Hospital between Jan 2015 and July 2019. Institutional ethical committee approval was obtained prior to the start of this study. We were advised that additional patient consent was not required as all ultrasound records were examined within the centre where it was undertaken and basic clinical data were collected using a standard clinical audit protocol.

\subsection{Ultrasound examination}

All ultrasound examinations were performed at the Fetal Medicine Research Institute 
110 using both transabdominal and transvaginal ultrasound transducers (GE Voluson ${ }^{\circledR} 730$,

111 GE Medical System, Zipf, Austria). The placenta was recorded as "low lying" when the

112 edge was $0.5-2 \mathrm{~cm}$ from the internal os of the uterine cervix. When the placenta was

$113<0.5 \mathrm{~cm}$ from the internal os or completely covering it, it was defined as placenta previa

114 (marginal or complete). For the review of ultrasound images, we used standardized

115 ultrasound descriptions and reporting pro-forma proposed by European Working Group

116 on Abnormally Invasive Placenta (EW-AIP) and the AIP international expert group [30].

117 For the comparison with the histopathology features, we recorded the following

118 ultrasound signs: loss of clear zone, myometrial thinning, placental lacunae (Fig 1A),

119 subplacental hypervascularity (Fig 1B) and lacunae feeder vessels. For the score of

120 placental lacunae, we used the classification proposed by Finberg and Williams $(0=$

121 none; $1+=1-3 ; 2+=4-6 ; 3+=>6$ ) [31]. All ultrasound images and video clips were

122 reviewed and agreed by two authors (NZ, EJ). The last ultrasound examination, which

123 was performed between 3 and 10 days before delivery, was used for the comparison

124 between ultrasound features and histological findings.

\subsection{Histopathologic examination}

127 Hysterectomy and myometrial resection specimens were sent to the pathology

128 department at King's College Hospital for gross examination in the fixed state and 129 dissection by the duty pathologist as per the Royal College of Pathologists guidelines 130 for the tissue pathways for gynaecological pathology (https://www.rcpath.org). As per

131 the local protocol for the gross examination of uterine specimens, the uterus was then 132 opened laterally and placed in $10 \%$ neutral buffered formalin. The site of accreta 
133 placentation was identified on gross examination as the lower anterior portion of the

134 uterine wall, corresponding to the previous caesarean section site (Fig 1C). samples $(2 \times 2 \times 0.5 \mathrm{~cm})$ including the whole thickness of the myometrium were taken from areas suspected of abnormal adherence or invasion (Fig 1D). Tissue blocks were paraffin embedded and sections stained with hematoxylin and eosin (H\&E) using routine methods. All slides were examined by a specialist perinatal pathologist $(\mathrm{HS})$, and the histology report documented the presence and grade of villous invasion. The presence or absence of decidua was assessed by cell morphology and the presence, the number and depth of villous invasion was determined using standard histological classification (3). Cases with large areas of missing decidua between the anchoring villi and the myometrium but with no invasive villi on any of the slides were recorded as placenta creta (adherenta). Placenta increta was recorded when the villi were found to invade the myometrium up to the uterine serosa and placenta percreta when the villi also invaded the uterine serosa and beyond in at least one slide. A second assessment was performed to identify vascular lesions i.e. fibrin deposition, intervillous thrombosis and villous infarction of at least half the placental tissue thickness. In slides where the full thickness of the myometrium was preserved $(n=187)$, the number of arteries within the superficial layer of myometrium immediately under the

152 basal plate were counted and described as fully transformed (full vascular 153 circumference replaced by fibrinoid with embedded endovascular trophoblast), partially 154 transformed (portion of the vessel wall lacked replacement by trophoblast) or untransformed. Instances of villi invading a vessel's wall and clusters of villi in the lumen 
156 of vessels were noted. Areas of definite myometrial scarring were identified by myofiber

157 disarray and tissue edema. At assessment, the assessor (EJ) was blinded to the ultrasound findings and original histological diagnosis of the cases.

\subsection{Immunohistochemistry}

161 Cytokeratin 7 (CK7) immunohistochemistry was used to complement H\&E analysis, by highlighting EVT cells in 55 samples with evidence of villous invasion. Briefly, sections 4-5 $\mu \mathrm{m}$ thick were deparaffinized and rehydrated, followed by antigen retrieval using citrate buffer. After being treated with $3 \%$ hydrogen peroxide to block endogenous peroxidase activity, the slides were incubated with mouse monoclonal antihuman CK7 antibody (M7018, Dako, CA, USA; 1:200 dilution). The reaction was developed with 3,3diaminobenzidine (DAB; Sigma Chemical Co.) containing $0.01 \% \mathrm{H}_{2} \mathrm{O}_{2}$ for $2-3$ min to give a brown reaction product. The sections were counterstained with Gills's no.2 hematoxylin (GH5232-IL, Sigma, London) for $30 \mathrm{~s}$, dehydrated, cleared in xylene and mounted with DPX synthetic resin (Raymond A. Lamb Ltd., London, UK). Positive controls were included in each staining run. Negative controls with replacement of the primary antibody by appropriate non-immune serum were performed for each sample. Distribution patterns of the CK7-positive EVTs were recorded as 'confluent' or 'scattered' as previously described [23]. In brief, EVTs were considered 'confluent' when 175 they formed diffuse sheet-like arrangements, continuous cell cords, or large clusters occupying more than two-thirds of the captured fields, and 'scattered' when they were distributed individually or formed small groups of five or fewer cells. 


\subsection{Statistical analysis}

StatGraphic-plus Version 3 data analysis and statistical software package (Manugistics, Rockville, MD) was used to analyse the data. A standard Kurtosis analysis indicated all values were normally distributed and the data are therefore presented as mean and standard deviation (SD). The data were separated into subgroups according to placental lacunae score and grade of placental adherence (creta) versus invasiveness (increta and percreta). Categorical variables were compared between subgroups using the Pearson's chi-square test. Continuous variables were analysed using a t-test to compare the means between the adherent and invasive subgroups and F-ratio for the analysis of variance (ANOVA) to compare the means between the lacunae subgroups. A $P$ value $<0.05$ was considered significant.

\section{Results}

\subsection{Clinical characteristics}

The patients ranged in age from 27 to 47 (mean (SD): 36.5 (4.1)) years, and the gestational ages at last scan and delivery ranged from 25 to 36 (mean (SD): 33.3 (2.6)) weeks and 29 to 37 (mean (SD): 35.3 (2.6)) weeks, respectively. The mean gravidity was 4.5 (SD 1.9) and the mean parity was 2.8 (SD 1.7). All 31 patients had a history of at least one prior caesarean delivery (mean (SD): $2.2(1.3)$ ). A primary caesarean hysterectomy was performed in 25 cases. The remaining six patients were delivered by fundal caesarean section followed partial myometrial resection of the accreta area and uterine preservation. In all cases, the fetuses were of appropriate growth for gestational age. 
3.2 Ultrasound imaging features

204 Three patients were diagnosed with an anterior low-lying placenta and 28 with an 205 anterior placenta previa, including eight with a marginal location and 20 partially or 206 completely covering the internal os of the cervix. Loss of clear zone and myometrial 207 thinning was observed under the placentation site in all cases. In seven cases, the myometrial thickness was $1-2 \mathrm{~mm}$ and was $<1 \mathrm{~mm}$ in the other cases.

At the last scan before delivery, all 31 patients presented with placental lacunae (Fig 1A) on the last ultrasound examination before delivery. Subplacental hypervascularity (Fig 1B) was found in 25 of the 31 cases, and lacunae feeder vessels were identified in nine cases. The lacunae score was 1+, 2+ and 3+ in 10, 11 and 10 cases, respectively.

In 14 cases, the patients had repeated ultrasound examinations at the Fetal Medicine Research Institute. In three of these cases lacunae were found at the first ultrasound examination at 11-14 weeks of gestation, and in the remaining 11 cases they were noted during the second trimester (20-28 weeks).

Tables 1 and 2 display and compare the main ultrasound features of PAS according to the lacunae score and grade of placental invasiveness as confirmed by histological examination, respectively. There were significant differences in the 221 distribution of subplacental vascularity, with ten out 11 cases with lacunae type 2+ and

222 all ten cases with lacunae $3+$ presenting with hypervascularity $(p<0.05)$. Similarly, all but 223 one of the invasive cases presented with hypervascularity $(p<0.005)$. Feeding vessels 224 were identified in nine cases, eight of which were associated with lacunae 3+ and one 
with lacunae 2+.

\subsection{Histopathologic examination and immunohistochemistry}

The initial histopathologic examination identified 22 cases with invasive villi in two or more slides (Fig 2), including two cases of placenta percreta with villi invading the whole thickness of the myometrium and the serosa. Areas where chorionic villi were in direct contact with the myometrium without interposing decidua basalis were found in both adherent (creta) and invasive cases (increta and percreta) (Fig 2B). In the latter, these areas were adjacent to the foci of invasive villi. Areas of villous invasion were characterized by variable and irregular villous ingrowth into the myometrium (Figs $2 \mathrm{~A}$ and $2 \mathrm{C}$ ), resulting in irregular, undulating basal plates with thick fibrin deposition surrounding all invasive villi (Fig 2D). In all the cases, placental villi displayed gestational age appropriate morphology and maturity. There were no trophoblast inclusions or fetal artery thromboses in any of the 31 cases, but a small placental infarct was found in one slide in two cases. Villi invading a uteroplacental artery wall were observed in one invasive case, whereas clusters of villi were found in the lumen of intramyometrial vessels in eight invasive cases (Fig 3A).

Large, recent intervillous thromboses in direct contact with the basal plate and extending to at least half of the placental tissue thickness (Fig 3B) were found in one adherent and 12 invasive cases (Table 3$)$. There were significant $(p<0.001$ and $p<0.05)$ differences in the distribution of these lesions in relation to both the lacunae scores and grade of villous invasiveness subgroups (Tables 1 and 2). Plaques of fibrin deposition were found in 14 of the 31 cases immediately above the basal plate (Fig 2A), but there 
was no significant difference between the subgroups.

Areas of definite myometrial scarring associated with zones of adherent or invasive villi were clearly demonstrated in the slides of 25 cases (Figs $2 \mathrm{~A}$ and $2 \mathrm{~B}$ ). Uteroplacental arteries with physiological change were found in all cases, with the proportion ranging between 5.6 and $84.6 \%$ of the arteries counted within the superficial layer of myometrium. Overall the mean proportions of partially and untransformed arteries were $20.04 \%$ (SD 9.17) and 32.74\% (SD 19.58), respectively. There was no significant difference in the mean count of partially remodeled vessels or vessels completely lacking remodeling according to the lacunae score or the grade of placental invasiveness (Tables 1 and 2). In four invasive cases, partial remodeling vascular remodeling was observed in the myometrium vessels towards the serosa. No lesions of arteriosclerosis or of acute atherosis were found in any of the vessels examined.

CK7 immunostaining showed the EVT cells were arranged in superficial confluent sheets in 18 samples (Fig 4A), in superficial irregular clusters in 24 samples and were scattered deep below the basal plate, reaching the serosal surface of the uterus in 13 samples (Fig 4B). In ten samples of the latter, the myometrium showed extended scarring. MNGCs were found in all cases with superficial layers EVT but not in samples with deep EVT invasion.

\section{Discussion}

Our study describes for the first time a direct relationship between the vascular changes observed on ultrasound imaging in PAS and histopathologic findings. The data indicate that villous invasion of the myometrium is associated with the development of placental 
lacunae on ultrasound and a high incidence of secondary vascular lesions seen within the intervillous space on histological examintion.

The main ultrasound signs used in the prenatal diagnosis of PAS are the loss of clear zone, myometrial thinning, the presence of lacunae and subplacental or uterovesical hypervascularity [7]. The clear zone describes the hypoechoic retroplacental zone in the myometrium under the placental bed, but its anatomical structure has not been firmly defined. It may correspond to the extensive vascular plexus and decidual glands under the placentation area between the tips of the anchoring villi and the junctional zone [23]. Its anatomy is subject to major changes in thickness as the decidua becomes thinner and the vascular plexus is compressed as pregnancy advances. We found that the clear zone was absent on the last ultrasound examination before delivery in all cases in this series.

Similarly, all cases presented with a thin lower uterine segment i.e. $<2 \mathrm{~mm}$ in thickness. The myometrial thickness was found to be less than $1 \mathrm{~mm}$ in 24 out of the 31 cases on ultrasound examination (Table 1) and was not related to the PAS grade (Table 2). Measurement of the myometrial thickness on ultrasound is not standardised in the diagnosis of PAS, and the thickness decreases physiologically in all pregnancies after 32 weeks when the lower uterine segment is further stretched by the combined action of the fetal presentation and Braxton-Hicks uterine contractions [32,33]. These anatomical changes will be more pronounced in patients who have had one or more prior caesarean deliveries as multiple lower segment scars often lead to scar defects and a large dehiscence at the end of pregnancy [34].

Placental lacunae are irregular sonolucent intra-placental spaces which have 
294 been reported $80 \%$ of the cohort studies independently of the depth of villous invasion

295 [7]. Placental lacunae were the first ultrasound sign reported on grey-scale imaging

296 suggestive of PAS. The presence of numerous lacunae i.e. $>6$, using the classification

297 proposed by Finberg and Williams [31] gives the placenta a "moth-eaten" appearance

298 typical of PAS on both transabdominal and transvaginal ultrasound examination (Fig

299 1A). Finberg and Williams also found a relationship between their lacunae score and the 300 grade of PAS with lacunae 3+ more often associated with placenta increta or percreta

301 [31]. Our data showing that all cases presenting with lacunae 3+ were found to have invasive villi on histopathologic examination support this finding (Table 1) and confirm placental lacunae as the primary sign indicating possible PAS on ultrasound imaging.

Previous immunohistochemistry studies have shown deeper EVT invasion and a higher number of EVT cells in the superficial myometrium of PAS cases [24-26,28]. As also shown in our study, EVT invasion patterns in PAS may vary and form confluent sheets, irregular clusters, or continuous cords [24-26]. The cords are thought to reflect preferential invasion along the plane of cleavage between adjacent bundles of muscle fibers. Both the distribution of spiral artery vascular remodelling and the depth and pattern of EVT invasion are likely to be influenced by the amount of scar tissue in the area of sampling. A more accurate evaluation could only be performed using sections of the entire placental bed in hysterectomy specimens.

Several authors have reported on the reduced degree of spiral artery remodeling in PAS $[21,25,26]$, but have not quantified their findings nor have they differentiated

315 between abnormally adherent and invasive cases or areas. In the present study, we 316 found that the proportion of fully transformed arteries ranged between 5.6 and $84.6 \%$ of 
317 the arteries counted in the superficial layer of myometrium, and that the mean proportions of partially and untransformed arteries were independent of the lacunae score and grade of PAS (Table 1 and 2). The lack of difference in fetal growth in pregnancies complicated by placenta previa accreta compared to low-lying and placenta previa with no evidence of PAS [35] suggest that these changes in uteroplacental vascular remodeling are focal and have little or no impact on overall placental biological functions. Like Khong et al, [21], we also found some degree of remodeling of the arteries in the deep myometrium. These findings support the concept that deeper than normal EVT invasion through the entire depth of the myometrium, with some degree of transformation of the deep arterial vasculature, leads to the vascular changes seen on CDI. The excessive dilatation of the radial, and in some cases of the arcuate, arteries in the accreta area can explain the development of lacunae and their feeding vessels. We have recently shown that excessive dilatation of the utero-placental circulation changes may be found away from the invasive area [36] and suggest that the extensive network of arterio-venous shunts found normally within the myometrium of the placental bed [37] may be involved. These findings can explain the area of hypervascularisation seen on CDI, and its association with lacunae 3+ score and villous invasion. The presence of clusters of chorionic villi in the lumens of myometrial vessels has been previously reported by Parra-Herran and Djordjevic [28] in invasive PAS. We found that these villi are of normal maturation and structure and are not attached to the vessel wall (Fig 3A). They are therefore probably secondary to embolization through arterio-venous shunts during the surgical procedure of caesarean hysterectomy. Our previous mathematical modelling of the transformation of the utero-placental 
340 circulation during the first half of pregnancy has shown the dramatic impact that the 341 terminal dilation of the arteries has on blood flow velocity [38]. Spiral artery remodelling

342 is essential to reduce the velocity from approximately $2-3 \mathrm{~m} / \mathrm{s}$ to around $10 \mathrm{~cm} / \mathrm{s}$ and 343 pressure with which the maternal arterial blood enters the intervillous space in order to 344 avoid damage to the villous trees. In invasive PAS, maternal blood from a radial or 345 arcuate artery will enter the intervillous space with a peak systolic velocity substantially 346 greater than $10 \mathrm{~cm} / \mathrm{sec}$ [23]. This distorts the anatomy of one or more lobules of the 347 early definitive placenta, including the corresponding inter-lobar septa [12] and leads to 348 the development of placental lacunae [23]. As shown in the present study, these 349 lacunae can be found from the beginning of the second trimester suggesting that these 350 areas of higher than normal blood velocity may chronically impact on the anatomy of the 351 villous tress and intervillous space.

We found thick basal plate fibrinoid deposits in all the areas of abnormally 353 adherent and invasive villous tissue (Fig 2). In 14 of our 31 cases there were also fibrin 354 deposition extending above the basal plate. The villous trophoblast is mechanically 355 sensitive [39]. Increased blood flow velocity and volume in areas with dense villous population such as those near the basal plate could result in chronic shear stress, which

357 could alter their apical membranes and lead to increased fibrin deposition. These

358 findings could explain the changes observed in the levels of maternal serum

359 biomarkers, such as human chorionic gonadotrophin (hCG) or alpha-fetoprotein (AFP) 360 in pregnancies presenting with PAS [40]. Large recent intervillous thromboses in direct 361 contact with the basal plate and extending into the villous tissue above (Fig 3B) were 362 found mainly in cases with lacunae $3+$ suggesting that these are the consequence of 
363 maternal blood thrombi formed within the lacunae space following the interruption of the 364 utero-placental circulation during the surgical procedure.

The main strength of our study is to have compared standardized ultrasound 366 imaging signs of PAS with detailed microscopic findings of the entire thickness of the 367 placentation area in hysterectomy specimens with placenta in-situ. This methodological 368 approach has allowed us to accurately correlate the deep myometrial and uteroplacental vascular changes associated with PAS and to differentiate between adherent and invasive cases. A limitation of modern histopathological studies on PAS is the lack of control group as primary caesarean hysterectomy with the placenta in-situ are now very rarely performed for indications other than invasive PAS. Thus, most data on placentation in normal pregnancies were obtained from historical hysterectomy 374 collections such as the Boyd \& Hamilton collection (The Centre for Trophoblast 375 Research, University of Cambridge). Using this collection, we found recently that in late second trimester hysterectomy specimens with no evidence of PAS, the decidual layer becomes thinner and discontinuous with advancing gestation [23]. This can also make the microscopic diagnosis of adherent placenta difficult, in particular if the accreta area of the myometrium is not extensively sampled. Attempts at manual removal of the placenta can distort the spatial relationship between the accreta villous tissue and the uterine wall [3]. This may limit the accuracy of the microscopic diagnosis in some cases,

382 in particular, if the histological examination of the utero-placental interface is incomplete. 383 In all cases of the present study, the diagnosis of PAS was made before delivery and 384 thus no attempt was made to remove the placenta during the delivery. 
1. E. Jauniaux, D. Ayres-de-Campos, J. Langhoff-Roos, K.A. Fox, S. Collins; FIGO Placenta Accreta Diagnosis and Management Expert Consensus Panel. FIGO classification for the clinical diagnosis of placenta accreta spectrum disorders, Int. J. Gynaecol. Obstet. 14 (2019) 20-24.

2. C. Irving, A.T. Hertig. A study of placenta accreta. Surgery, Gynecol. Obstet. 64 (1937) 178-200.

3. R.K. Luke, J.W. Sharpe, R.R. Greene. Placenta accreta: The adherent or invasive placenta, Am. J. Obstet. Gynecol. 95 (1966) 660-668.

4. E. Jauniaux, F. Chantraine, R.M. Silver, J. Langhoff-Roos; FIGO Placenta Accreta Diagnosis and Management Expert Consensus Panel. FIGO consensus guidelines on placenta accreta spectrum disorders: Epidemiology, Int. J. Gynaecol. Obstet. 140 (2018) 265-273.

5. Y. Gielchinsky, N. Rojansky, S.J. Fasouliotis, Y. Ezra. Placenta accreta-summary of 10 years: a survey of 310 cases. Placenta. 23 (2002) 210-214.

6. T. Michikawa, S. Morokuma, S. Yamazaki, K. Fukushima, K. Kato, H. Nitta. Exposure to air pollutants during the early weeks of pregnancy, and placenta praevia and placenta accreta in the western part of Japan, Environ. Int. 92 (2016) 464-470.

7. E. Jauniaux, S.L. Collins, D. Jurkovic, G.J. Burton. Accreta placentation. A systematic review of prenatal ultrasound imaging and grading of villous invasiveness, Am. J. Obstet. Gynecol. 215 (2016) 712-721. 
8. E. Jauniaux, C. Bunce, L. Grønbeck, J. Langhoff-Roos. Prevalence and main outcomes of placenta accreta spectrum: a systematic review and metaanalysis, Am. J. Obstet. Gynecol. 221 (2019) 208-218.

9. E. Jauniaux, L. Grønbeck, C. Bunce, J. Langhoff-Roos, S.L. Collins. Epidemiology of placenta previa accreta: a systematic review and meta-analysis, B.M.J. Open. 9 (2019) e031193.

10. E. Jauniaux, D. Jurkovic. Placenta accreta: pathogenesis of a 20th century iatrogenic uterine disease, Placenta. 33 (2012) 244-251.

11. H.J. Baldwin, J.A. Patterson, T.A. Nippita, S. Torvaldsen, I. Ibiebele, J.M. Simpson, J.B. Ford. Antecedents of abnormally invasive placenta in primiparous women: risk associated with gynecologic procedures, Obstet. Gynecol. 131 (2018) 227-233.

12. H. Fox. Placenta accreta: 1945-1969, Obstet, Gynecol, Survey. 27 (1972) 475490.

13. N. Zosmer, J. Fuller, H. Shaikh, J. Johns, J.A. Ross. Natural history of early firsttrimester pregnancies implanted in Cesarean scars, Ultrasound Obstet. Gynecol. 46 (2015) 367-375.

14. G. Cali, F. Forlani, I.E. Timor-Tritsch, J. Palacios-Jaraquemada, G. Minneci, F. D'Antonio. Natural history of Cesarean scar pregnancy on prenatal ultrasound: the crossover sign, Ultrasound Obstet. Gynecol. 50 (2017) 100-104.

15. E. Jauniaux, A. Bhide. Prenatal ultrasound diagnosis and outcome of placenta previa accreta after caesarean delivery: a systematic review and meta-analysis, Am. J. Obstet. Gynecol. 217 (2017) 27-36. 
16.E. Jauniaux, G.J. Burton. Pathophysiology of placenta accreta spectrum disorders: A review of current finding, Clin. Obstet. Gynecol. 61 (2018) 743-754.

17.H.A. Roeder, S.F. Cramer, P.C. Leppert. A look at uterine wound healing through a histopathological study of uterine scars, Reprod. Sci. 19 (2012) 463-473.

18. R. Pijnenborg, L. Vercruysse, I. Brosens. Deep placentation, Best. Pract. Research Clin. Obstet .Gynaecol. 25 (2011) 273-285.

19. I. Brosens, P. Puttemans, G. Benagiano. Placental bed research: I. The placental bed: from spiral arteries remodeling to the great obstetrical syndromes, Am. J. Obstet. Gynecol. 221 (2019) 437-456.

20. R.S. Al-Lamki, J.N. Skepper, G.J. Burton. Are human placental bed giant cells merely aggregates of small mononuclear trophoblast cells? An ultrastructural and immunocytochemical study, Hum. Reprod. 14 (1999) 496-504.

21. T.Y. Khong, W.B. Robertson. Placenta creta and placenta praevia creta, Placenta. 8 (1987) 399-409.

22. K.M. Tabsh, C.R. 3rd Brinkman, W. King. Ultrasound diagnosis of placenta increta, J. Clin. Ultrasound. 10 (1982) 288-290.

23. E. Jauniaux, S.L. Collins, G.J. Burton. Placenta accreta spectrum:

Pathophysiology and evidence-based anatomy for prenatal ultrasound imaging, Am. J Obstet. Gynecol. 218 (2018) 75-87.

24. K.R. Kim, S.Y. Jun, J.Y. Kim, J.Y. Ro. Implantation site intermediate trophoblast in placenta cretas, Mod. Pathol. 17 (2004) 1483-1490.

25. P. Tantbirojn, C.P. Crum, M.M. Parast. Pathophysiology of placenta creta: the role of decidua and extravillous trophoblast, Placenta. 29 (2008) 639-645. 
26. T. Hannon, B.A. Innes, G.E. Lash J.N. Bulmer, S.C. Robson. Effects of local decidua on trophoblast invasion and spiral artery remodeling in focal placenta creta - an immunohistochemical study, Placenta. 33 (2012) 998-1004.

27.E. Adler, R. Madankumar, M. Rosner, S.E. Reznik. Increased placental trophoblast inclusions in placenta accreta, Placenta. 35 (2014) 10751078.

28. C. Parra-Herran, B. Djordjevic. Histopathology of placenta creta: chorionic villi intrusion into myometrial vascular spaces and extravillous trophoblast proliferation are frequent and specific findings with implications on diagnosis and pathogenesis, Int. J. Gynecol .Pathol. 35 (2016) 497-508.

29. K. Dannheim, S.A. Shainker, J.L. Hecht. Hysterectomy for placenta accreta; methods for gross and microscopic pathology examination, Arch. Gynecol. Obstet. 293 (2016) 951-958.

30.S.L. Collins, A. Ashcroft, T. Braun, P. Calda, J Langhoff-Ross, O. Morel, V Stefanovic, B. Tutschek, F. Chantraine. Proposal for standardized ultrasound descriptions of abnormally invasive placenta (AIP), Ultrasound Obstet. Gynecol. 47 (2016) 271-275.

31. H.J. Finberg, J.W. Williams. Placenta accreta: prospective sonographic diagnosis in patients with placenta previa and prior cesarean section, J. Ultrasound Med. 11 (1992) 333-343.

32. N. Kok, I.C. Wiersma, B.C. Opmeer, I.M. de Graaf, B.W. Mol, E. Pajkrt. Sonographic measurement of lower uterine segment thickness to predict uterine 
rupture during a trial of labor in women with previous Cesarean section: a metaanalysis. Ultrasound Obstet. Gynecol. 42 (2013) 132-139.

33. A. Baranov, K.Å. Salvesen, O. Vikhareva. Validation of prediction model for successful vaginal birth after Cesarean delivery based on sonographic assessment of hysterotomy scar. Ultrasound Obstet Gynecol. 51 (2018) 189-193.

34.E. Jauniaux, A.M. Hussein, K.A. Fox, S.L. Collins. New evidence-based diagnostic and management strategies for placenta accreta spectrum disorders, Best Pract. Res. Clin. Obstet. Gynaecol. 61 (2019) 75-88.

35. E. Jauniaux, I. Dimitrova, N. Kenyon, M. Mhallem, N.A. Kametas, N. Zosmer, C. Hubinont, K.H. Nicolaides, S.L. Collins. Impact of placenta previa with placenta accreta spectrum disorder on fetal growth, Ultrasound Obstet. Gynecol. 54 (2019) 643-649.

36. E. Jauniaux, A.M. Hussein, N. Zosmer, R.M. Elbarmelgy, R.A. Elbarmelgy, H. Shaikh, G.J. Burton. A new methodologic approach for clinico-pathologic correlations in invasive placenta previa accreta, Am. J. Obstet. Gynecol. 222 (2020) 379.e1-379.e11.

37. J.P. Schaaps, V. Tsatsaris, F. Goffin, J.F. Brichant, K. Delbecque, M. Tebache, L. Collignon, M.C. Retz, J.M. Foidart. Shunting the intervillous space: new concepts in human uteroplacental vascularization, Am. J. Obstet. Gynecol. 192 (2005) 323-332.

38.G.J. Burton, A.W. Woods, E. Jauniaux, J.C. Kingdom. Rheological and physiological consequences of conversion of the maternal spiral arteries for uteroplacental blood flow during human pregnancy, Placenta. 30 (2009) 473-482. 
39. L.C. Morley, D.J. Beech, J.J. Walker, N.A.B. Simpson. Emerging concepts of shear stress in placental development and function, Mol. Hum. Reprod. 25 (2019) 329-339.

40. H.C. Bartels, J.D. Postle, P. Downey, D.J. Brennan. Placenta Accreta Spectrum: A Review of pathology, molecular biology, and biomarkers, Dis. Markers. 2018 (2018):1507674. 


\section{1}

2

\title{
Ultrasound-histopathologic features of the utero-placental interface in placenta accreta spectrum
}

\author{
Eric Jauniaux ${ }^{a}$, Nurit Zosmer ${ }^{\text {b }}$, Devi Subramanian ${ }^{b}$, Hizbullah Shaikh ${ }^{c}$, Graham J \\ Burton $^{\text {d }}$ \\ ${ }^{\text {a} E G A ~ I n s t i t u t e ~ f o r ~ W o m e n ' s ~ H e a l t h, ~ F a c u l t y ~ o f ~ P o p u l a t i o n ~ H e a l t h ~ S c i e n c e s, ~}$ \\ University College London (UCL), London, UK. \\ 'The Fetal Medicine Research Institute, Harris Birthright Research Centre, King's \\ College Hospital, London, UK \\ 'Department of Histopathology (Dr Shaikh), King's College Hospital, London, UK \\ dThe Centre for Trophoblast Research (CTR), Department of Physiology, \\ Development and Neuroscience, University of Cambridge, Cambridge, United \\ Kingdom.
}

Conflict of interest: none

Word count main text: 3740

No funding was obtained for this study.

Corresponding author: Professor Eric Jauniaux, Institute for Women's Health, University College London, 86-96 Chenies Mews, London WC1E 6HX, UK.

Telephone numbers: $+44 / 207 / 3908113$

Fax: +44/207/3908115

E-mail: e.jauniaux@ucl.ac.uk 


\section{Introduction}

Placenta accreta spectrum (PAS) is a modern disorder of human placentation which is graded according to the degree of villous adherence to, or invasion into, the uterine wall by the placental tissue [1]. Until the end of the last century, PAS was a very rare complication of pregnancy, affecting $<1$ in 10,000 livebirths [2,3]. Following the exponential increase in the number of caesarean deliveries during the last two decades, the prevalence of PAS has risen to $<1$ in 1,000 livebirths [4] with some authors reporting prevalence as high as $1 \%$ of livebirths in their population $[5,6]$. Epidemiological data on prevalence of PAS are highly heterogenous due to the lack of a standardised terminology [7] and confirmation of the degree of villous invasion at birth in most case series $[8,9]$.

Any form of damage, even small, to the integrity of the endometrial uterine lining following a uterine curettage, myomectomy, or endometrial resection has been associated with the development of both adherent and invasive accreta placentation in subsequent pregnancies [10-12]. However, in the majority of these cases there was no evidence of myometrial invasion. Similarly, in the first detailed case series of accreta placentation reported in 1937 by Irving and Hertig [2] all 18 cases were described as abnormally adherent with no macroscopic nor histological evidence of myometrial villous invasion. By contrast, the current trend of multiple caesarean deliveries often leads to the development of large and deep myometrial defects, and there is a direct association between blastocyst implantation in a caesarean scar and the development of placenta previa accreta $[13,14]$. More than $90 \%$ of cases of PAS are now found in women with a history of prior caesarean delivery presenting with a low-lying or placenta 
previa [15], suggesting a tropism of the blastocyst for the scar area [16]. In contrast to uterine epithelial layers that heal by regeneration and recolonization of scar areas, the myometrium, does not heal by regenerating muscle fibers and myometrial scar tissue often presents with myofibre disarray, tissue edema, inflammation and elastosis [17]. These data support the concept that in PAS the trophoblast is biologically normal but becomes excessively invasive secondary to implantation into an anatomically abnormal uterine bed resulting from a surgical scar $[10,16]$.

Placentation in the human in physiologically invasive [18]. In normal pregnancies, the interstitial extravillous trophoblast (EVT) invade the uterine wall as far as the inner third of the myometrium [19], also called the junctional-zone (JZ), where they fuse to form multinucleated trophoblast giant cells (MNGCs) [20]. In the weeks following implantation, EVT cells are found both within and around the spiral arteries in the central area of the placental bed. Both endovascular and interstitial EVT invasion are associated with the physiological conversion of the terminal part of the uterine circulation down to the level of the spiral arteries in the $\mathrm{JZ}$ of the inner third of the myometrium. In PAS the EVT cells are increased in size and number, and the depth of their myometrial invasion is greater [21]. There is also a failure of fusion of the EVT to form MNGCs. As a result, the EVT come into contact with, and invade into the walls of, the radial and even the arcuate arteries.

Tabsh et al. [22] in 1982 were the first to report on the prenatal diagnosis of a case of placenta increta using grey-scale ultrasound imaging. The use of colour-Doppler imaging (CDI) two decades later has enabled a better visualisation of the vascular changes associated with PAS before birth [7]. Increased sub-placental hypervascularity 
and the presence of intra-placental lacunae are the most prominent features of invasive PAS prenatally on ultrasound [23]. Only a few authors have recently reported on the histopathology of the placenta and utero-placental interface in PAS [24-29], but none have studied the possible link between prenatal ultrasound features and placental pathologic findings. Similarly, authors of ultrasound imaging cohort studies have rarely provided detailed histopathologic findings beyond the classical description of absent decidua or the presence of invasive villi on histologic examination [7-9]. The purpose of our study was twofold: first to evaluate the impact of the changes in the utero-placental and intervillous circulations seen on ultrasound imaging on histopathologic features in PAS, and secondly to compare the extensiveness of EVT migration and uterine arterial transformation according to the grade of villous invasion.

\section{Material and Methods}

Our study group included 31 patients with singleton pregnancies diagnosed prenatally with low-lying/placenta previa accreta and confirmed by histopathology. All patients delivered at King's College Hospital between Jan 2015 and July 2019. Institutional ethical committee approval was obtained prior to the start of this study. We were advised that additional patient consent was not required as all ultrasound records were examined within the centre where it was undertaken and basic clinical data were collected using a standard clinical audit protocol.

\subsection{Ultrasound examination}

All ultrasound examinations were performed at the Fetal Medicine Research Institute 
110 using both transabdominal and transvaginal ultrasound transducers (GE Voluson ${ }^{\circledR} 730$,

111 GE Medical System, Zipf, Austria). The placenta was recorded as "low lying" when the

112 edge was $0.5-2 \mathrm{~cm}$ from the internal os of the uterine cervix. When the placenta was

$113<0.5 \mathrm{~cm}$ from the internal os or completely covering it, it was defined as placenta previa

114 (marginal or complete). For the review of ultrasound images, we used standardized

115 ultrasound descriptions and reporting pro-forma proposed by European Working Group

116 on Abnormally Invasive Placenta (EW-AIP) and the AIP international expert group [30].

117 For the comparison with the histopathology features, we recorded the following

118 ultrasound signs: loss of clear zone, myometrial thinning, placental lacunae (Fig 1A),

119 subplacental hypervascularity (Fig 1B) and lacunae feeder vessels. For the score of

120 placental lacunae, we used the classification proposed by Finberg and Williams $(0=$

121 none; $1+=1-3 ; 2+=4-6 ; 3+=>6$ ) [31]. All ultrasound images and video clips were

122 reviewed and agreed by two authors (NZ, EJ). The last ultrasound examination, which

123 was performed between 3 and 10 days before delivery, was used for the comparison

124 between ultrasound features and histological findings.

\subsection{Histopathologic examination}

127 Hysterectomy and myometrial resection specimens were sent to the pathology

128 department at King's College Hospital for gross examination in the fixed state and 129 dissection by the duty pathologist as per the Royal College of Pathologists guidelines 130 for the tissue pathways for gynaecological pathology (https://www.rcpath.org). As per

131 the local protocol for the gross examination of uterine specimens, the uterus was then 132 opened laterally and placed in $10 \%$ neutral buffered formalin. The site of accreta 
133 placentation was identified on gross examination as the lower anterior portion of the uterine wall, corresponding to the previous caesarean section site (Fig 1C).

After formalin fixation for a minimum of 6-7 days, between five and seven samples $(2 \times 2 \times 0.5 \mathrm{~cm})$ including the whole thickness of the myometrium were taken from areas suspected of abnormal adherence or invasion (Fig 1D). Tissue blocks were paraffin embedded and sections stained with hematoxylin and eosin (H\&E) using routine methods. All slides were examined by a specialist perinatal pathologist $(\mathrm{HS})$, and the histology report documented the presence and grade of villous invasion. The presence or absence of decidua was assessed by cell morphology and the presence, the number and depth of villous invasion was determined using standard histological classification (3). Cases with large areas of missing decidua between the anchoring villi and the myometrium but with no invasive villi on any of the slides were recorded as placenta creta (adherenta). Placenta increta was recorded when the villi were found to invade the myometrium up to the uterine serosa and placenta percreta when the villi also invaded the uterine serosa and beyond in at least one slide. A second assessment was performed to identify vascular lesions i.e. fibrin deposition, intervillous thrombosis and villous infarction of at least half the placental tissue thickness.

In slides where the full thickness of the myometrium was preserved $(n=187)$, the number of arteries within the superficial layer of myometrium immediately under the basal plate were counted and described as fully transformed (full vascular circumference replaced by fibrinoid with embedded endovascular trophoblast), partially transformed (portion of the vessel wall lacked replacement by trophoblast) or untransformed. Instances of villi invading a vessel's wall and clusters of villi in the lumen 
156 of vessels were noted. Areas of definite myometrial scarring were identified by myofiber

157 disarray and tissue edema. At assessment, the assessor (EJ) was blinded to the

158 ultrasound findings and original histological diagnosis of the cases.

\subsection{Immunohistochemistry}

161 Cytokeratin 7 (CK7) immunohistochemistry was used to complement H\&E analysis, by highlighting EVT cells in 55 samples with evidence of villous invasion. Briefly, sections 4-5 $\mu \mathrm{m}$ thick were deparaffinized and rehydrated, followed by antigen retrieval using citrate buffer. After being treated with $3 \%$ hydrogen peroxide to block endogenous peroxidase activity, the slides were incubated with mouse monoclonal antihuman CK7 antibody (M7018, Dako, CA, USA; 1:200 dilution). The reaction was developed with 3,3diaminobenzidine (DAB; Sigma Chemical Co.) containing $0.01 \% \mathrm{H}_{2} \mathrm{O}_{2}$ for $2-3$ min to give a brown reaction product. The sections were counterstained with Gills's no.2 hematoxylin (GH5232-IL, Sigma, London) for $30 \mathrm{~s}$, dehydrated, cleared in xylene and mounted with DPX synthetic resin (Raymond A. Lamb Ltd., London, UK). Positive controls were included in each staining run. Negative controls with replacement of the primary antibody by appropriate non-immune serum were performed for each sample. Distribution patterns of the CK7-positive EVTs were recorded as 'confluent' or 'scattered' as previously described [23]. In brief, EVTs were considered 'confluent' when 175 they formed diffuse sheet-like arrangements, continuous cell cords, or large clusters occupying more than two-thirds of the captured fields, and 'scattered' when they were distributed individually or formed small groups of five or fewer cells. 


\subsection{Statistical analysis}

StatGraphic-plus Version 3 data analysis and statistical software package (Manugistics, Rockville, MD) was used to analyse the data. A standard Kurtosis analysis indicated all values were normally distributed and the data are therefore presented as mean and standard deviation (SD). The data were separated into subgroups according to placental lacunae score and grade of placental adherence (creta) versus invasiveness (increta and percreta). Categorical variables were compared between subgroups using the Pearson's chi-square test. Continuous variables were analysed using a t-test to compare the means between the adherent and invasive subgroups and F-ratio for the analysis of variance (ANOVA) to compare the means between the lacunae subgroups. A $P$ value $<0.05$ was considered significant.

\section{Results}

\subsection{Clinical characteristics}

The patients ranged in age from 27 to 47 (mean (SD): 36.5 (4.1)) years, and the gestational ages at last scan and delivery ranged from 25 to 36 (mean (SD): 33.3 (2.6)) weeks and 29 to 37 (mean (SD): 35.3 (2.6)) weeks, respectively. The mean gravidity was 4.5 (SD 1.9) and the mean parity was 2.8 (SD 1.7). All 31 patients had a history of at least one prior caesarean delivery (mean (SD): $2.2(1.3)$ ). A primary caesarean hysterectomy was performed in 25 cases. The remaining six patients were delivered by fundal caesarean section followed partial myometrial resection of the accreta area and uterine preservation. In all cases, the fetuses were of appropriate growth for gestational age. 


\subsection{Ultrasound imaging features}

204 Three patients were diagnosed with an anterior low-lying placenta and 28 with an 205 anterior placenta previa, including eight with a marginal location and 20 partially or 206 completely covering the internal os of the cervix. Loss of clear zone and myometrial 207 thinning was observed under the placentation site in all cases. In seven cases, the myometrial thickness was $1-2 \mathrm{~mm}$ and was $<1 \mathrm{~mm}$ in the other cases.

At the last scan before delivery, all 31 patients presented with placental lacunae (Fig 1A) on the last ultrasound examination before delivery. Subplacental were identified in nine cases. The lacunae score was $1+, 2+$ and $3+$ in 10, 11 and 10 cases, respectively. Medicine Research Institute. In three of these cases lacunae were found at the first were noted during the second trimester (20-28 weeks).

Tables 1 and 2 display and compare the main ultrasound features of PAS according to the lacunae score and grade of placental invasiveness as confirmed by histological examination, respectively. There were significant differences in the distribution of subplacental vascularity, with ten out 11 cases with lacunae type $2+$ and all ten cases with lacunae $3+$ presenting with hypervascularity $(p<0.05)$. Similarly, all but one of the invasive cases presented with hypervascularity $(p<0.005)$. Feeding vessels were identified in nine cases, eight of which were associated with lacunae 3+ and one 
with lacunae 2+.

\subsection{Histopathologic examination and immunohistochemistry}

The initial histopathologic examination identified 22 cases with invasive villi in two or more slides (Fig 2), including two cases of placenta percreta with villi invading the whole thickness of the myometrium and the serosa. Areas where chorionic villi were in direct contact with the myometrium without interposing decidua basalis were found in both adherent (creta) and invasive cases (increta and percreta) (Fig 2B). In the latter, these areas were adjacent to the foci of invasive villi. Areas of villous invasion were characterized by variable and irregular villous ingrowth into the myometrium (Figs $2 \mathrm{~A}$ and $2 \mathrm{C}$ ), resulting in irregular, undulating basal plates with thick fibrin deposition surrounding all invasive villi (Fig 2D). In all the cases, placental villi displayed gestational age appropriate morphology and maturity. There were no trophoblast inclusions or fetal artery thromboses in any of the 31 cases, but a small placental infarct was found in one slide in two cases. Villi invading a uteroplacental artery wall were observed in one invasive case, whereas clusters of villi were found in the lumen of intramyometrial vessels in eight invasive cases (Fig 3A).

Large, recent intervillous thromboses in direct contact with the basal plate and extending to at least half of the placental tissue thickness (Fig 3B) were found in one adherent and 12 invasive cases (Table 3$)$. There were significant $(p<0.001$ and $p<0.05)$ differences in the distribution of these lesions in relation to both the lacunae scores and grade of villous invasiveness subgroups (Tables 1 and 2). Plaques of fibrin deposition were found in 14 of the 31 cases immediately above the basal plate (Fig 2A), but there 
was no significant difference between the subgroups.

Areas of definite myometrial scarring associated with zones of adherent or invasive villi were clearly demonstrated in the slides of 25 cases (Figs $2 A$ and $2 B$ ). Uteroplacental arteries with physiological change were found in all cases, with the proportion ranging between 5.6 and $84.6 \%$ of the arteries counted within the superficial layer of myometrium. Overall the mean proportions of partially and untransformed arteries were $20.04 \%$ (SD 9.17) and 32.74\% (SD 19.58), respectively. There was no significant difference in the mean count of partially remodeled vessels or vessels completely lacking remodeling according to the lacunae score or the grade of placental invasiveness (Tables 1 and 2). In four invasive cases, partial remodeling vascular remodeling was observed in the myometrium vessels towards the serosa. No lesions of arteriosclerosis or of acute atherosis were found in any of the vessels examined.

CK7 immunostaining showed the EVT cells were arranged in superficial confluent sheets in 18 samples (Fig 4A), in superficial irregular clusters in 24 samples and were scattered deep below the basal plate, reaching the serosal surface of the uterus in 13 samples (Fig 4B). In ten samples of the latter, the myometrium showed extended scarring. MNGCs were found in all cases with superficial layers EVT but not in samples with deep EVT invasion.

\section{Discussion}

Our study describes for the first time a direct relationship between the vascular changes observed on ultrasound imaging in PAS and histopathologic findings. The data indicate that deep villous invasion of the myometrium is associated with the development of 
271 placental lacunae on ultrasound and a high incidence of secondary vascular lesions

272 seen within the intervillous space on histological examintionexamination.

The main ultrasound signs used in the prenatal diagnosis of PAS are the loss of clear zone, myometrial thinning, the presence of lacunae and subplacental or uterovesical hypervascularity [7]. The clear zone describes the hypoechoic retroplacental

276 zone in the myometrium under the placental bed, but its anatomical structure has not 277 been firmly defined. It may correspond to the extensive vascular plexus and decidual 278 glands under the placentation area between the tips of the anchoring villi and the 279 junctional zone [23]. Its anatomy is subject to major changes in thickness as the decidua becomes thinner and the vascular plexus is compressed as pregnancy advances. We found that the clear zone was absent on the last ultrasound examination before delivery in all cases in this series.

Similarly, all cases presented with a thin lower uterine segment i.e. $<2 \mathrm{~mm}$ in thickness. The myometrial thickness was found to be less than $1 \mathrm{~mm}$ in 24 out of the 31 cases on ultrasound examination (Table 1) and was not related to the PAS grade (Table 2). Measurement of the myometrial thickness on ultrasound is not standardised in the diagnosis of PAS, and the thickness decreases physiologically in all pregnancies after 32 weeks when the lower uterine segment is further stretched by the combined action of the fetal presentation and Braxton-Hicks uterine contractions [32,33]. These anatomical changes will be more pronounced in patients who have had one or more prior-previous caesarean deliveries as multiple lower segment scars often lead to scar defects and a

292 large dehiscence at the end of pregnancy [34]. 
294 been reported $80 \%$ of the cohort studies independently of the depth of villous invasion

295 [7]. Placental lacunae were the first ultrasound sign reported on grey-scale imaging

296 suggestive of PAS. The presence of numerous lacunae i.e. $>6$, using the classification

297 proposed by Finberg and Williams [31] gives the placenta a "moth-eaten" appearance

298 typical of PAS on both transabdominal and transvaginal ultrasound examination (Fig

299 1A). Finberg and Williams also found a relationship between their lacunae score and the

300 grade of PAS with lacunae 3+ more often associated with placenta increta or percreta

301 [31]. Our data showing that all cases presenting with lacunae 3+ were found to have

302 invasive villi on histopathologic examination support this finding (Table 1) and confirm

303 placental lacunae as the primary sign indicating possible PAS on ultrasound imaging.

304 Previous immunohistochemistry studies have shown deeper EVT invasion and a

305 higher number of EVT cells in the superficial myometrium of PAS cases [24-26,28]. As

306 also shown in our study, EVT invasion patterns in PAS may vary and form confluent

307 sheets, irregular clusters, or continuous cords [24-26]. The cords are thought to reflect

308 preferential invasion along the plane of cleavage between adjacent bundles of muscle

309 fibers. Both the distribution of spiral artery vascular remodelling and the depth and

310 pattern of EVT invasion are likely to be influenced by the amount of scar tissue in the

311 area of sampling. A more accurate evaluation could only be performed using sections of

312 the entire placental bed in hysterectomy specimens.

313 Several authors have reported on the reduced degree of spiral artery remodeling

314 in PAS $[21,25,26]$, but have not quantified their findings nor have they differentiated

315 between abnormally adherent and invasive cases or areas. In the present study, we

316 found that the proportion of fully transformed arteries ranged between 5.6 and $84.6 \%$ of 
317 the arteries counted in the superficial layer of myometrium, and that the mean proportions of partially and untransformed arteries were independent of the lacunae score and grade of PAS (Table 1 and 2). The lack of difference in fetal growth in pregnancies complicated by placenta previa accreta compared to low-lying and placenta previa with no evidence of PAS [35] suggest that these changes in uteroplacental vascular remodeling are focal and have little or no impact on overall placental biological functions. Like Khong et al, [21], we also found some degree of remodeling of the arteries in the deep myometrium. These findings support the concept that deeper seen on CDI.

The excessive dilatation of the radial, and in some cases of the arcuate, arteries in the accreta area can explain the development of lacunae and their feeding vessels. We have recently shown that excessive dilatation of the utero-placental circulation changes may be found away from the invasive area [36] and suggest that the extensive network of arterio-venous shunts found normally within the myometrium of the placental presence of clusters of chorionic villi in the lumens of myometrial vessels has been previously reported by Parra-Herran and Djordjevic [28] in invasive PAS. We found that bed [37] may be involved. These findings can explain the area of hypervascularisation seen on $\mathrm{CDI}$, and its association with lacunae 3+ score and villous invasion. The these villi are of normal maturation and structure and are not attached to the vessel wall (Fig 3A). They are therefore probably secondary to embolization through arterio-venous shunts during the surgical procedure of caesarean hysterectomy. 
Our previous mathematical modelling of the transformation of the utero-placental circulation during the first half of pregnancy has shown the dramatic impact that the terminal dilation of the arteries has on blood flow velocity [38]. Spiral artery remodelling is essential to reduce the velocity from approximately $2-3 \mathrm{~m} / \mathrm{s}$ to around $10 \mathrm{~cm} / \mathrm{s}$ and pressure with which the maternal arterial blood enters the intervillous space in order to avoid damage to the villous trees. In invasive PAS, maternal blood from a radial or arcuate artery will enter the intervillous space with a peak systolic velocity substantially greater than $10 \mathrm{~cm} / \mathrm{sec}$ [23]. This distorts the anatomy of one or more lobules of the early definitive placenta, including the corresponding inter-lobar septa [12] and leads to the development of placental lacunae [23]. As shown in the present study, these lacunae can be found from the beginning of the second trimester suggesting that these areas of higher than normal blood velocity may chronically impact on the anatomy of the villous tress and intervillous space.

We found thick basal plate fibrinoid deposits in all the areas of abnormally adherent and invasive villous tissue (Fig 2). In 14 of our 31 cases there were also fibrin deposition extending above the basal plate. The villous trophoblast is mechanically sensitive [39]. Increased blood flow velocity and volume in areas with dense villous population such as those near the basal plate could result in chronic shear stress, which could alter their apical membranes and lead to increased fibrin deposition. These findings could explain the changes observed in the levels of maternal serum biomarkers, such as human chorionic gonadotrophin (hCG) or alpha-fetoprotein (AFP) in pregnancies presenting with PAS [40]. Large recent intervillous thromboses in direct contact with the basal plate and extending into the villous tissue above (Fig 3B) were 
363 found mainly in cases with lacunae $3+$ suggesting that these are the consequence of

maternal blood thrombi formed within the lacunae space following the interruption of the utero-placental circulation during the surgical procedure.

The main strength of our study is to have compared standardized ultrasound imaging signs of PAS with detailed microscopic findings of the entire thickness of the placentation area in hysterectomy specimens with placenta in-situ. This methodological approach has allowed us to accurately correlate the deep myometrial and uteroplacental vascular changes associated with PAS and to differentiate between adherent and invasive cases. A limitation of modern histopathological studies on PAS is the lack of control group as primary caesarean hysterectomy with the placenta in-situ are now very rarely performed for indications other than invasive PAS. Thus, most data on placentation in normal pregnancies were obtained from historical hysterectomy collections such as the Boyd \& Hamilton collection (The Centre for Trophoblast Research, University of Cambridge). Using this collection, we found recently that in late second trimester hysterectomy specimens with no evidence of PAS, the decidual layer becomes thinner and discontinuous with advancing gestation [23]. This can also make the microscopic diagnosis of adherent placenta difficult, in particular if the accreta area of the myometrium is not extensively sampled. Attempts at manual removal of the placenta can distort the spatial relationship between the accreta villous tissue and the uterine wall [3]. This may limit the accuracy of the microscopic diagnosis in some cases, in particular, if the histological examination of the utero-placental interface is incomplete. In all cases of the present study, the diagnosis of PAS was made before delivery and thus no attempt was made to remove the placenta during the delivery. 
386 


\section{References}

1. E. Jauniaux, D. Ayres-de-Campos, J. Langhoff-Roos, K.A. Fox, S. Collins; FIGO Placenta Accreta Diagnosis and Management Expert Consensus Panel. FIGO classification for the clinical diagnosis of placenta accreta spectrum disorders, Int. J. Gynaecol. Obstet. 14 (2019) 20-24.

2. C. Irving, A.T. Hertig. A study of placenta accreta. Surgery, Gynecol. Obstet. 64 (1937) 178-200.

3. R.K. Luke, J.W. Sharpe, R.R. Greene. Placenta accreta: The adherent or invasive placenta, Am. J. Obstet. Gynecol. 95 (1966) 660-668.

4. E. Jauniaux, F. Chantraine, R.M. Silver, J. Langhoff-Roos; FIGO Placenta Accreta Diagnosis and Management Expert Consensus Panel. FIGO consensus guidelines on placenta accreta spectrum disorders: Epidemiology, Int. J. Gynaecol. Obstet. 140 (2018) 265-273.

5. Y. Gielchinsky, N. Rojansky, S.J. Fasouliotis, Y. Ezra. Placenta accreta-summary of 10 years: a survey of 310 cases. Placenta. 23 (2002) 210-214.

6. T. Michikawa, S. Morokuma, S. Yamazaki, K. Fukushima, K. Kato, H. Nitta. Exposure to air pollutants during the early weeks of pregnancy, and placenta praevia and placenta accreta in the western part of Japan, Environ. Int. 92 (2016) 464-470.

7. E. Jauniaux, S.L. Collins, D. Jurkovic, G.J. Burton. Accreta placentation. A systematic review of prenatal ultrasound imaging and grading of villous invasiveness, Am. J. Obstet. Gynecol. 215 (2016) 712-721. 
8. E. Jauniaux, C. Bunce, L. Grønbeck, J. Langhoff-Roos. Prevalence and main outcomes of placenta accreta spectrum: a systematic review and metaanalysis, Am. J. Obstet. Gynecol. 221 (2019) 208-218.

9. E. Jauniaux, L. Grønbeck, C. Bunce, J. Langhoff-Roos, S.L. Collins. Epidemiology of placenta previa accreta: a systematic review and meta-analysis, B.M.J. Open. 9 (2019) e031193.

10.E. Jauniaux, D. Jurkovic. Placenta accreta: pathogenesis of a 20th century iatrogenic uterine disease, Placenta. 33 (2012) 244-251.

11. H.J. Baldwin, J.A. Patterson, T.A. Nippita, S. Torvaldsen, I. Ibiebele, J.M. Simpson, J.B. Ford. Antecedents of abnormally invasive placenta in primiparous women: risk associated with gynecologic procedures, Obstet. Gynecol. 131 (2018) 227-233.

12. H. Fox. Placenta accreta: 1945-1969, Obstet, Gynecol, Survey. 27 (1972) 475490.

13. N. Zosmer, J. Fuller, H. Shaikh, J. Johns, J.A. Ross. Natural history of early firsttrimester pregnancies implanted in Cesarean scars, Ultrasound Obstet. Gynecol. 46 (2015) 367-375.

14. G. Cali, F. Forlani, I.E. Timor-Tritsch, J. Palacios-Jaraquemada, G. Minneci, F. D'Antonio. Natural history of Cesarean scar pregnancy on prenatal ultrasound: the crossover sign, Ultrasound Obstet. Gynecol. 50 (2017) 100-104.

15. E. Jauniaux, A. Bhide. Prenatal ultrasound diagnosis and outcome of placenta previa accreta after caesarean delivery: a systematic review and meta-analysis, Am. J. Obstet. Gynecol. 217 (2017) 27-36. 
16.E. Jauniaux, G.J. Burton. Pathophysiology of placenta accreta spectrum disorders: A review of current finding, Clin. Obstet. Gynecol. 61 (2018) 743-754.

17.H.A. Roeder, S.F. Cramer, P.C. Leppert. A look at uterine wound healing through a histopathological study of uterine scars, Reprod. Sci. 19 (2012) 463-473.

18. R. Pijnenborg, L. Vercruysse, I. Brosens. Deep placentation, Best. Pract. Research Clin. Obstet .Gynaecol. 25 (2011) 273-285.

19. I. Brosens, P. Puttemans, G. Benagiano. Placental bed research: I. The placental bed: from spiral arteries remodeling to the great obstetrical syndromes, Am. J. Obstet. Gynecol. 221 (2019) 437-456.

20. R.S. Al-Lamki, J.N. Skepper, G.J. Burton. Are human placental bed giant cells merely aggregates of small mononuclear trophoblast cells? An ultrastructural and immunocytochemical study, Hum. Reprod. 14 (1999) 496-504.

21. T.Y. Khong, W.B. Robertson. Placenta creta and placenta praevia creta, Placenta. 8 (1987) 399-409.

22. K.M. Tabsh, C.R. 3rd Brinkman, W. King. Ultrasound diagnosis of placenta increta, J. Clin. Ultrasound. 10 (1982) 288-290.

23. E. Jauniaux, S.L. Collins, G.J. Burton. Placenta accreta spectrum:

Pathophysiology and evidence-based anatomy for prenatal ultrasound imaging, Am. J Obstet. Gynecol. 218 (2018) 75-87.

24. K.R. Kim, S.Y. Jun, J.Y. Kim, J.Y. Ro. Implantation site intermediate trophoblast in placenta cretas, Mod. Pathol. 17 (2004) 1483-1490.

25. P. Tantbirojn, C.P. Crum, M.M. Parast. Pathophysiology of placenta creta: the role of decidua and extravillous trophoblast, Placenta. 29 (2008) 639-645. 
26. T. Hannon, B.A. Innes, G.E. Lash J.N. Bulmer, S.C. Robson. Effects of local decidua on trophoblast invasion and spiral artery remodeling in focal placenta creta - an immunohistochemical study, Placenta. 33 (2012) 998-1004.

27. E. Adler, R. Madankumar, M. Rosner, S.E. Reznik. Increased placental trophoblast inclusions in placenta accreta, Placenta. 35 (2014) 10751078.

28. C. Parra-Herran, B. Djordjevic. Histopathology of placenta creta: chorionic villi intrusion into myometrial vascular spaces and extravillous trophoblast proliferation are frequent and specific findings with implications on diagnosis and pathogenesis, Int. J. Gynecol .Pathol. 35 (2016) 497-508.

29. K. Dannheim, S.A. Shainker, J.L. Hecht. Hysterectomy for placenta accreta; methods for gross and microscopic pathology examination, Arch. Gynecol. Obstet. 293 (2016) 951-958.

30.S.L. Collins, A. Ashcroft, T. Braun, P. Calda, J Langhoff-Ross, O. Morel, V Stefanovic, B. Tutschek, F. Chantraine. Proposal for standardized ultrasound descriptions of abnormally invasive placenta (AIP), Ultrasound Obstet. Gynecol. 47 (2016) 271-275.

31. H.J. Finberg, J.W. Williams. Placenta accreta: prospective sonographic diagnosis in patients with placenta previa and prior cesarean section, J. Ultrasound Med. 11 (1992) 333-343.

32. N. Kok, I.C. Wiersma, B.C. Opmeer, I.M. de Graaf, B.W. Mol, E. Pajkrt. Sonographic measurement of lower uterine segment thickness to predict uterine 
rupture during a trial of labor in women with previous Cesarean section: a metaanalysis. Ultrasound Obstet. Gynecol. 42 (2013) 132-139.

33. A. Baranov, K.Å. Salvesen, O. Vikhareva. Validation of prediction model for successful vaginal birth after Cesarean delivery based on sonographic assessment of hysterotomy scar. Ultrasound Obstet Gynecol. 51 (2018) 189-193.

34.E. Jauniaux, A.M. Hussein, K.A. Fox, S.L. Collins. New evidence-based diagnostic and management strategies for placenta accreta spectrum disorders, Best Pract. Res. Clin. Obstet. Gynaecol. 61 (2019) 75-88.

35. E. Jauniaux , I. Dimitrova, N. Kenyon, M. Mhallem, N.A. Kametas, N. Zosmer, C. Hubinont, K.H. Nicolaides, S.L. Collins. Impact of placenta previa with placenta accreta spectrum disorder on fetal growth, Ultrasound Obstet. Gynecol. 54 (2019) 643-649.

36. E. Jauniaux, A.M. Hussein, N. Zosmer, R.M. Elbarmelgy, R.A. Elbarmelgy, H. Shaikh, G.J. Burton. A new methodologic approach for clinico-pathologic correlations in invasive placenta previa accreta, Am. J. Obstet. Gynecol. 222 (2020) 379.e1-379.e11.

37. J.P. Schaaps, V. Tsatsaris, F. Goffin, J.F. Brichant, K. Delbecque, M. Tebache, L. Collignon, M.C. Retz, J.M. Foidart. Shunting the intervillous space: new concepts in human uteroplacental vascularization, Am. J. Obstet. Gynecol. 192 (2005) 323-332.

38.G.J. Burton, A.W. Woods, E. Jauniaux, J.C. Kingdom. Rheological and physiological consequences of conversion of the maternal spiral arteries for uteroplacental blood flow during human pregnancy, Placenta. 30 (2009) 473-482. 
39. L.C. Morley, D.J. Beech, J.J. Walker, N.A.B. Simpson. Emerging concepts

501 of shear stress in placental development and function, Mol. Hum. Reprod. 25

502 (2019) 329-339.

40. H.C. Bartels, J.D. Postle, P. Downey, D.J. Brennan. Placenta Accreta Spectrum: A Review of pathology, molecular biology, and biomarkers, Dis. Markers. 2018

505 (2018):1507674.

506

507 


\section{Figure legends}

Fig 1. Transvaginal ultrasound views of a placenta $(P)$ increta showing in $A$ : numerous large lacunae (stage $3+$ ) and in B: increased sub-placental hypervascularity and intra-lacunar blood flow on CDI; C: Anterior view of the hysterectomy specimens showing the fundal caesarean section (CS) incision. On opening, the placenta was previa covering the entire lower segment (LS) and the cervix confirming the ultrasound diagnosis; D: Histological section (H\&E x 0.8) of the myometrium $(\mathrm{M})$ under the increta area of the placenta $(\mathrm{P})$.

Fig 2. A: Full-thickness section of the uterine wall under adherent villi to the basal plate $(B P)$ without interposing decidua (H\&E x 1.5). Note the myofiber disarray and tissue edema of the underlying myometrium $(\mathrm{M})$ and the presence of large vessels (V); B: Section of the myometrium under an invasive area (H\&E x 2.0). Invasive villi surrounded by the irregular, undulating basal plate (BP) with thick fibrin deposition (FB) surrounding invasive anchoring villi; C: Deep villous invasion area (arrows) (H\&E x 2.9); D: Invasive villi separated from the edematous myometrium (M) by thick fibrin deposition (FD) (H\&E x 10.0).

Fig 3. A: Cluster of chorionic villi (CV) in the lumen of a transformed intramyometrial vessel (H\&E x 10.0); B: Large recent intervillous thrombosis (IVT) in direct contact with the basal plate (BP) next to an invasive area (arrow). The IVT extends to at least half of the placental tissue thickness and the BP normal structure under the IVT is disrupted by extensive fibrin deposition and the uterine wall is very thin and made of scar tissue (H\&E x 2.5).

Fig 4. CK7 staining showing EVT cells arranged in confluent sheets $(A)$ or scattered deep (B) below the basal plate (BP). 
Table 1. Distribution of the ultrasound signs and histological features according to the placental lacunae classification [30].

\begin{tabular}{|c|c|c|c|c|}
\hline Variables & $\begin{array}{l}\text { Lacunae } 1+ \\
n=10\end{array}$ & $\begin{array}{c}\text { Lacunae } 2+ \\
n=11\end{array}$ & $\begin{array}{c}\text { Lacunae } 3+ \\
n=10\end{array}$ & $p$ \\
\hline \multicolumn{5}{|l|}{ ULTRASOUND } \\
\hline $\begin{array}{c}\text { Myometrial thickness } \\
-\quad 1-2 \mathrm{~mm} \\
-\quad<1 \mathrm{~mm} \\
\end{array}$ & $\begin{array}{l}2 \\
8\end{array}$ & $\begin{array}{l}2 \\
9\end{array}$ & $\begin{array}{l}3 \\
7 \\
\end{array}$ & $0.7887^{*}$ \\
\hline $\begin{aligned} & \text { Subplacental vascularity } \\
&- \text { Normal } \\
&- \text { Increased (HV) }\end{aligned}$ & $\begin{array}{l}5 \\
5\end{array}$ & $\begin{array}{c}1 \\
10\end{array}$ & $\begin{array}{c}0 \\
10\end{array}$ & $0.0103^{*}$ \\
\hline \multicolumn{5}{|l|}{ HISTOLOGY } \\
\hline $\begin{array}{cc}\text { PAS grade } \\
- & \text { Adherent } \\
- & \text { Invasive } \\
\end{array}$ & $\begin{array}{l}6 \\
4 \\
\end{array}$ & $\begin{array}{l}3 \\
8\end{array}$ & $\begin{array}{c}0 \\
10\end{array}$ & $0.0125^{\star}$ \\
\hline $\begin{aligned} & \text { Intervillous thrombosis } \\
&- \text { No } \\
&- \text { Yes }\end{aligned}$ & $\begin{array}{l}8 \\
2\end{array}$ & $\begin{array}{l}9 \\
2\end{array}$ & $\begin{array}{l}1 \\
9\end{array}$ & $0.0009^{*}$ \\
\hline $\begin{array}{cc}\text { Fibrin deposition } \\
-\quad \text { Basal plate } \\
-\quad \text { Extended }\end{array}$ & $\begin{array}{l}6 \\
4\end{array}$ & $\begin{array}{l}7 \\
4 \\
\end{array}$ & $\begin{array}{l}4 \\
6\end{array}$ & $0.5116^{*}$ \\
\hline $\begin{array}{l}\text { Mean (SD) superficial } \\
\text { myometrium arterial } \\
\text { transformation } \\
\text { - } \quad \text { Partial } \\
\text { - } \quad \text { Not transformed }\end{array}$ & $\begin{array}{c}18.9(8.1) \\
28.9(14.7)\end{array}$ & $\begin{array}{c}18.9(8.1) \\
28.56(14.7)\end{array}$ & $\begin{array}{l}21.6(10.1) \\
36.6(18.9)\end{array}$ & $\begin{array}{l}0.7995^{\#} \\
0.6858^{\#}\end{array}$ \\
\hline
\end{tabular}

$\mathrm{HV}=$ hypervascularity

${ }^{*}$ Chi-square test; ${ }^{\#}$ F-ratio (ANOVA) 
Table 2. Distribution of the ultrasound signs and histological features according to grade of villous invasiveness.

\section{Histological grade}

\begin{tabular}{|c|c|c|c|}
\hline Variables & $\begin{array}{c}\text { Adherent } \\
n=9\end{array}$ & $\begin{array}{c}\text { Invasive } \\
n=22\end{array}$ & $p$ \\
\hline ULTRASOUND & & & \\
\hline $\begin{array}{c}\text { Myometrial thickness } \\
-\quad 1-2 \mathrm{~mm} \\
-\quad<1 \mathrm{~mm}\end{array}$ & $\begin{array}{l}2 \\
7\end{array}$ & $\begin{array}{c}5 \\
17\end{array}$ & $0.9757^{*}$ \\
\hline $\begin{array}{cl}\text { Subplacental vascularity } \\
-\quad & \text { Normal } \\
-\quad & \text { Increased (HV) }\end{array}$ & $\begin{array}{l}5 \\
4\end{array}$ & $\begin{array}{c}1 \\
21\end{array}$ & $0.0011^{*}$ \\
\hline HISTOLOGY & & & \\
\hline $\begin{array}{c}\text { Intervillous thrombosis } \\
-\quad \text { No } \\
-\quad \text { Yes }\end{array}$ & $\begin{array}{l}8 \\
1\end{array}$ & $\begin{array}{l}10 \\
12\end{array}$ & $0.0261^{*}$ \\
\hline $\begin{array}{c}\text { Fibrin deposition } \\
-\quad \text { Basal plate } \\
-\quad \text { Extended }\end{array}$ & $\begin{array}{l}7 \\
2\end{array}$ & $\begin{array}{l}10 \\
12\end{array}$ & $0.1007^{*}$ \\
\hline $\begin{array}{l}\text { Mean (SD) superficial } \\
\text { myometrium arterial } \\
\text { transformation } \\
-\quad \text { Partial } \\
-\quad \text { Not transformed }\end{array}$ & $\begin{array}{c}18.2(6.0) \\
25.8(13.5)\end{array}$ & $\begin{array}{l}20.7(10.2) \\
35.6(21.2)\end{array}$ & $\begin{array}{l}0.4958^{\#} \\
0.2129^{\#}\end{array}$ \\
\hline
\end{tabular}

$\mathrm{HV}=$ hypervascularity

${ }^{*}$ Chi-square test; ${ }^{\#}$ t-test; 
Fig 2. A: Full-thickness section of the uterine wall under adherent villi to the basal plate $(B P)$ without interposing decidua (H\&E x 1.5). Note the myofiber disarray and tissue edema of the underlying myometrium $(\mathrm{M})$ and the presence of large vessels (V); B: Section of the myometrium under an invasive area (H\&E x 2.0). Invasive villi surrounded by the irregular, undulating basal plate (BP) with thick fibrin deposition (FB) surrounding invasive anchoring villi; C: Deep villous invasion area (arrows) (H\&E x 2.9); D: Invasive villi separated from the edematous myometrium (M) by thick fibrin deposition (FD) (H\&E x 10.0).

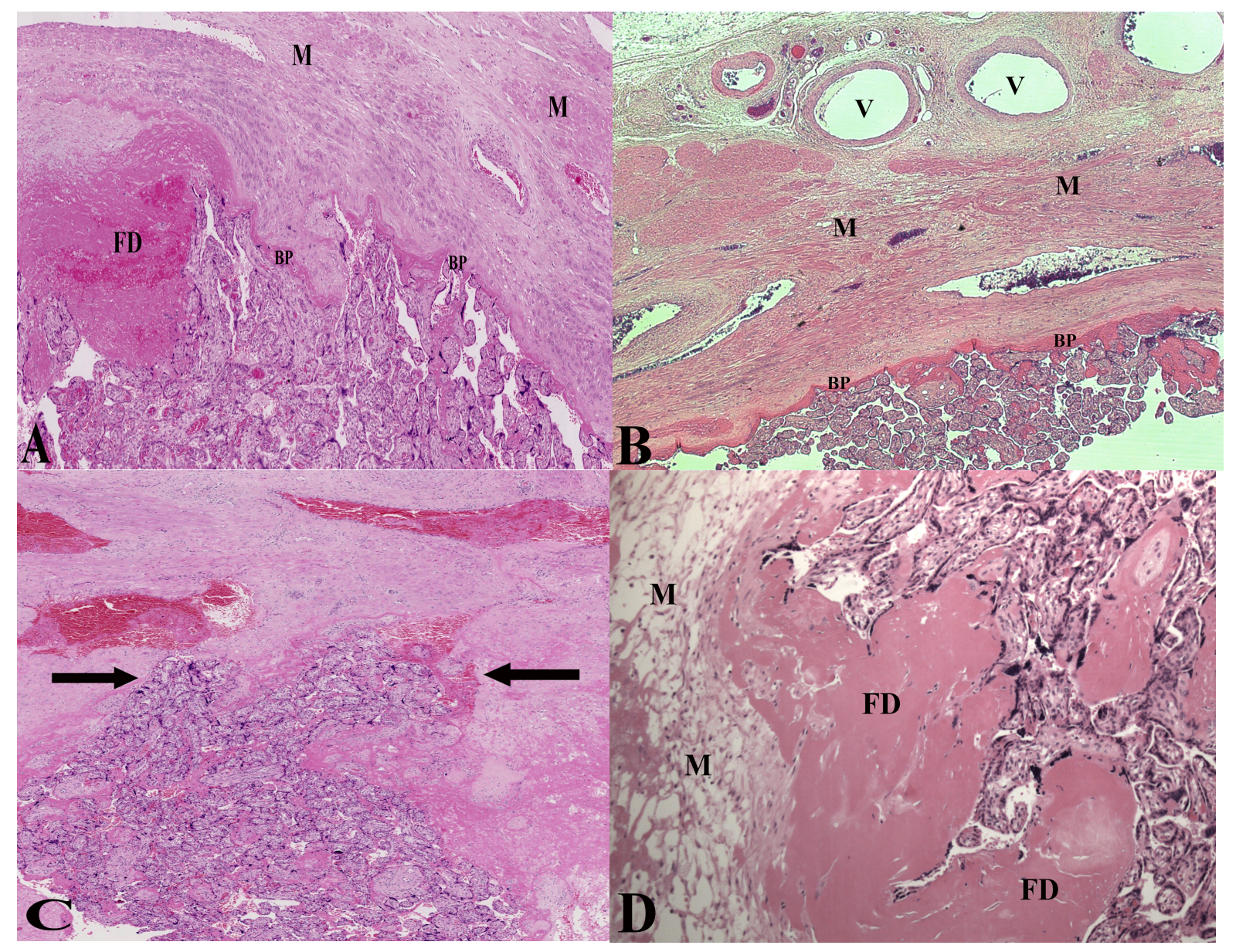


Fig 3. A: Cluster of chorionic villi (CV) in the lumen of a transformed intramyometrial vessel (H\&E x 10.0); B: Large recent intervillous thrombosis (IVT) in direct contact with the basal plate (BP) next to an invasive area (arrow). The IVT extends to at least half of the placental tissue thickness and the BP normal structure under the IVT is disrupted by extensive fibrin deposition and the uterine wall is very thin and made of scar tissue (H\&E x 2.5).

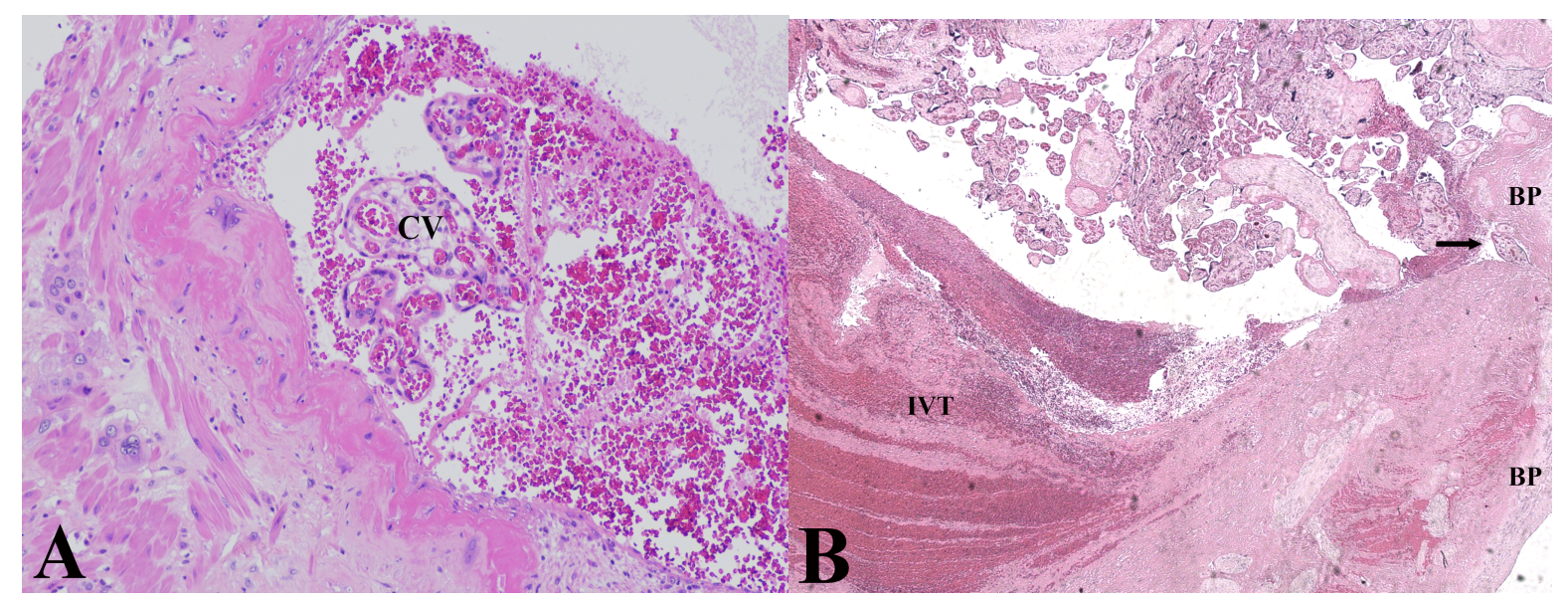


Fig 4. CK7 staining showing EVT cells arranged in confluent sheets $(\mathbf{A})$ or scattered deep (B) below the basal plate (BP).

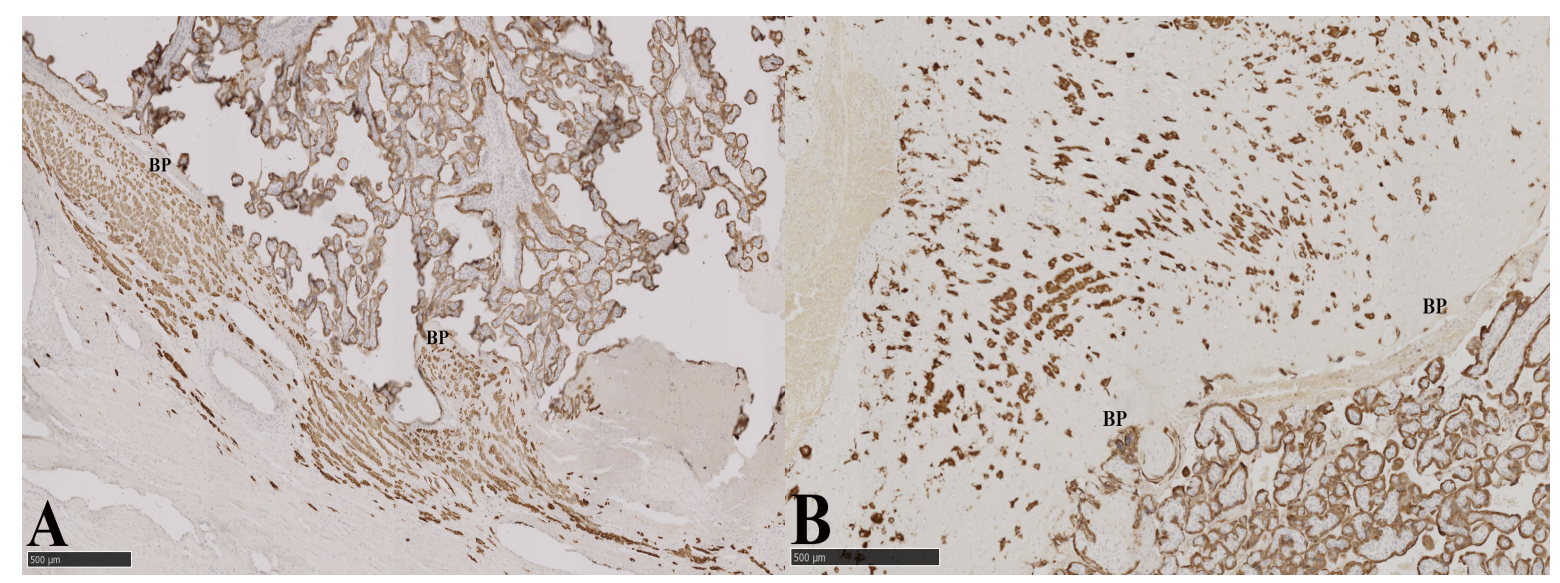




\section{Figure legends}

Fig 1. Transvaginal ultrasound views of a placenta $(P)$ increta showing in $A$ : numerous large lacunae (stage $3+$ ) and in B: increased sub-placental hypervascularity and intra-lacunar blood flow on CDI; C: Anterior view of the hysterectomy specimens showing the fundal caesarean section (CS) incision. On opening the placenta seen to be previa covering the entire lower segment (LS) and the cervix confirming the ultrasound diagnosis; D: Histological section (H\&E x 0.8) of the myometrium $(\mathrm{M})$ under the increta area of the placenta $(P)$.
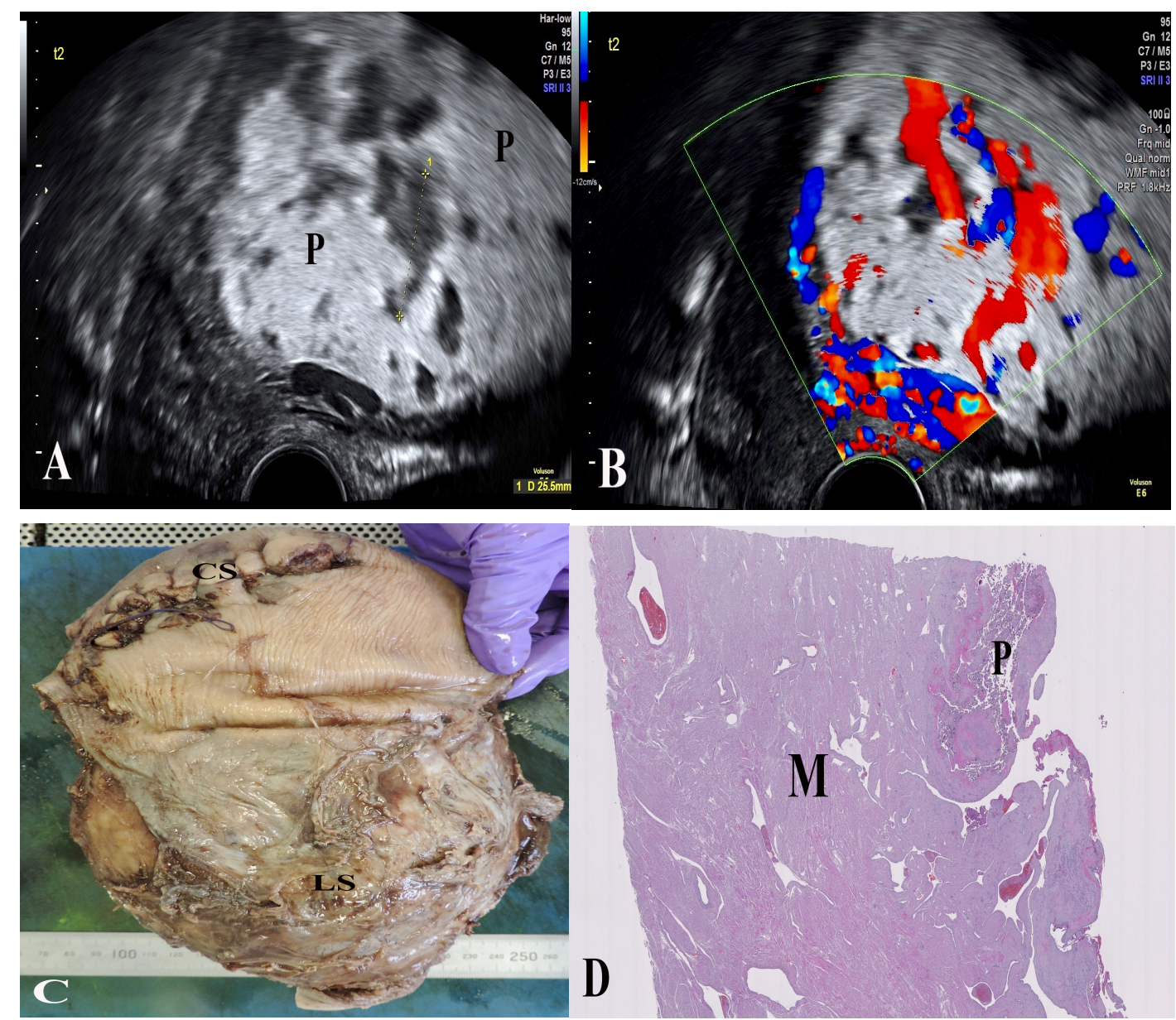
Click here to download high resolution image

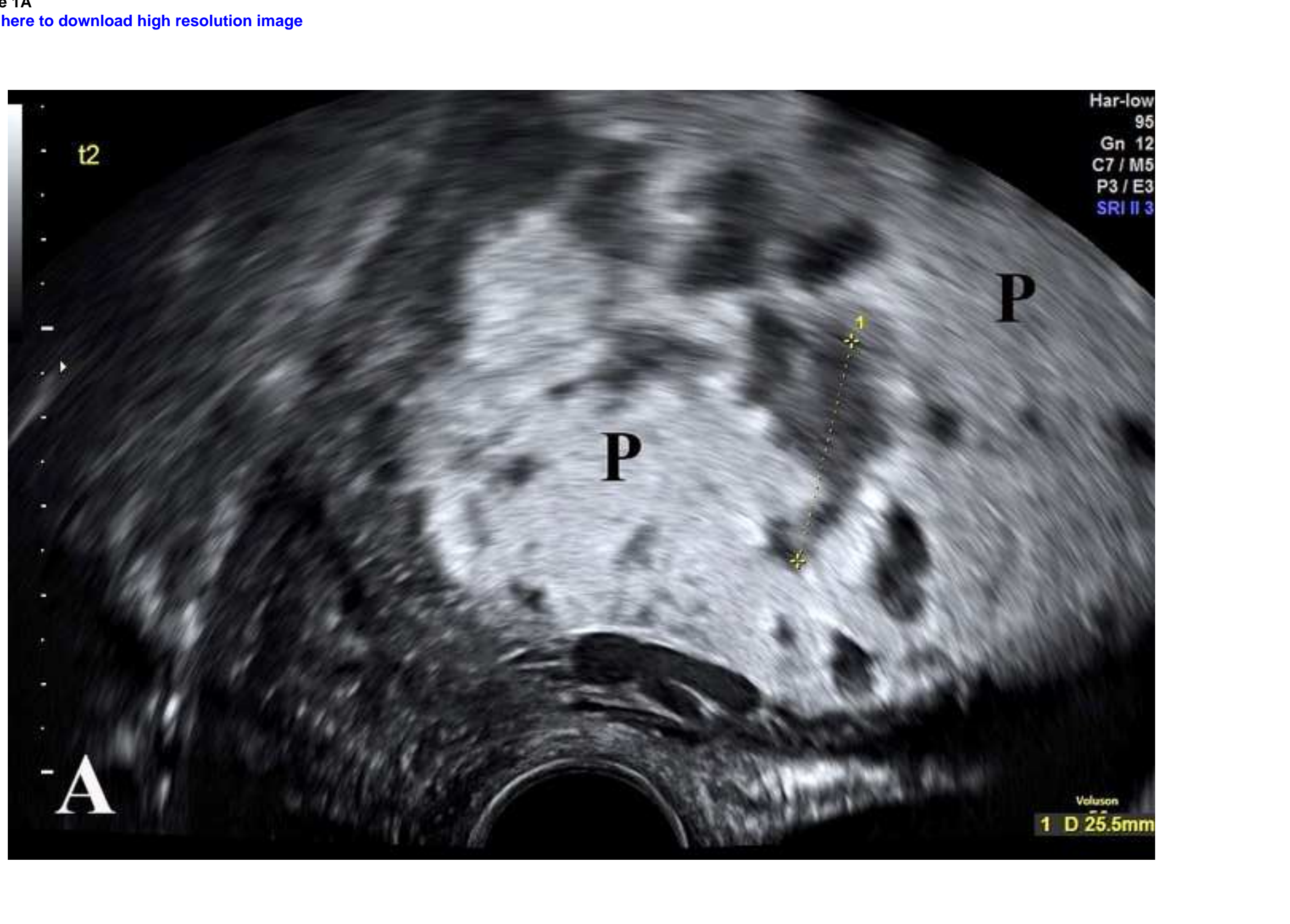

.
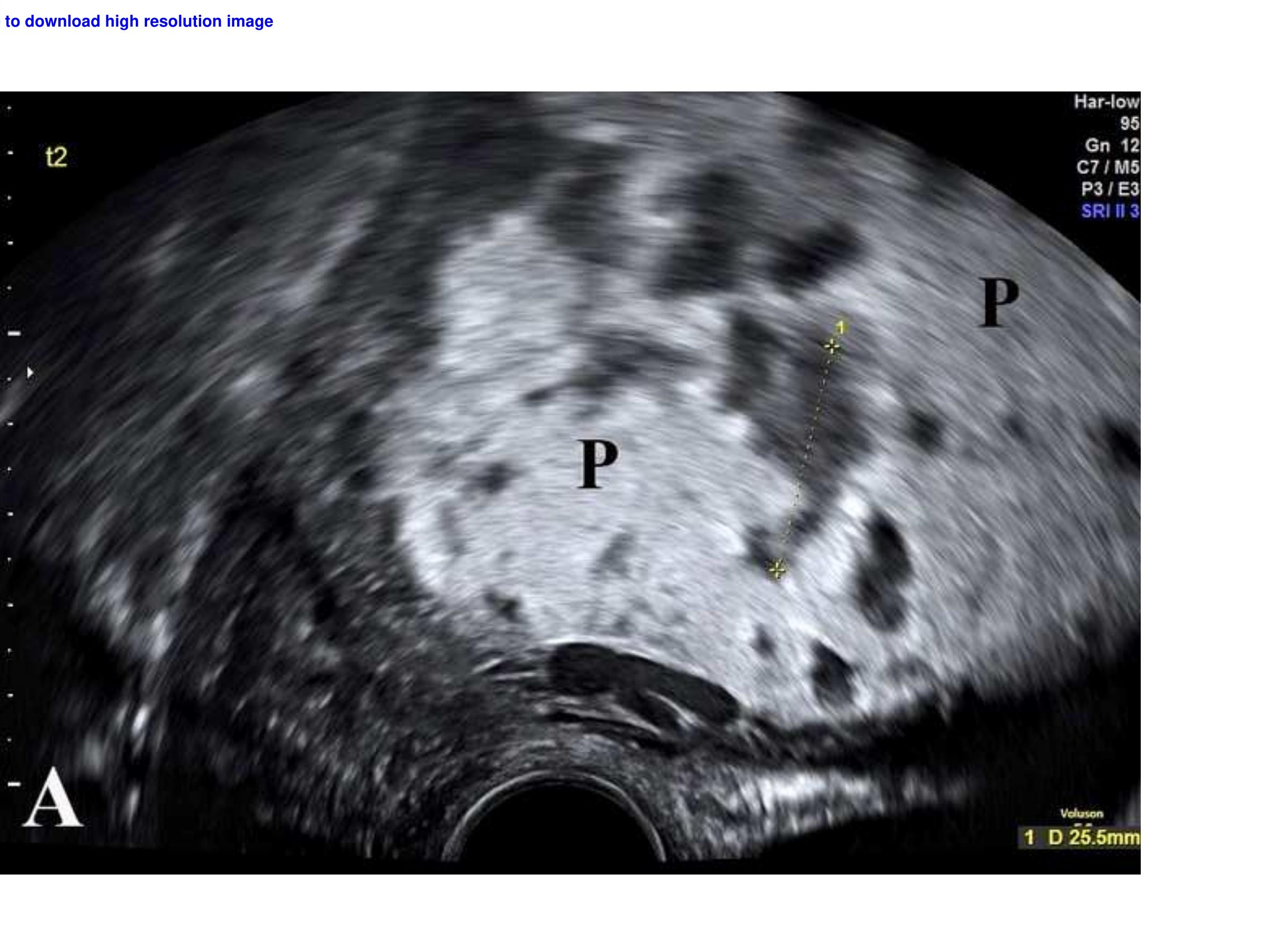
Figure 1B
Click here to download high resolution image

Figure 1B
Click here to download high resolution image

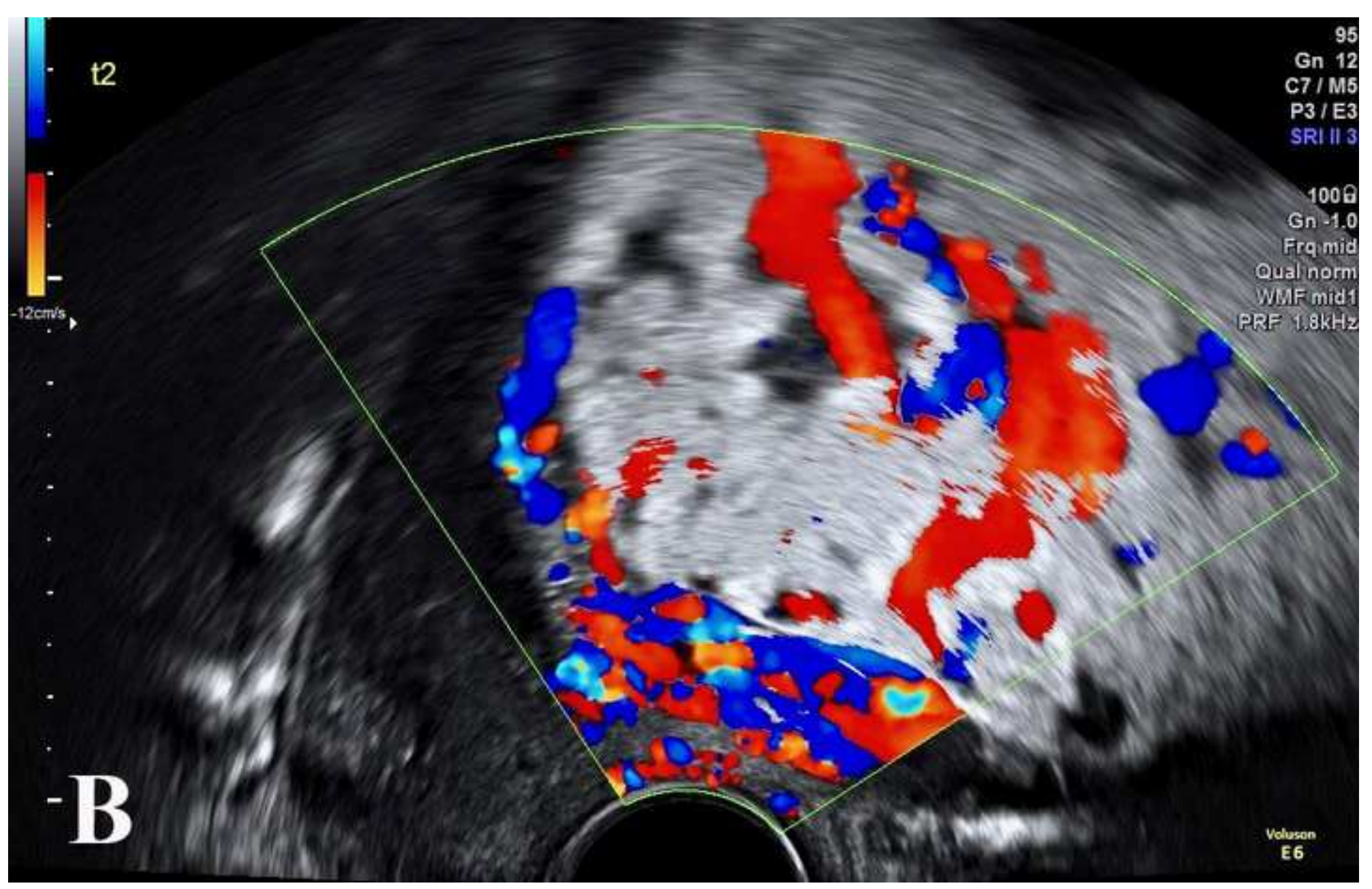

\section{,}

I M5

$3 / \mathrm{E} 3$

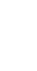

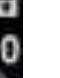
. .

(1)

(1)

.




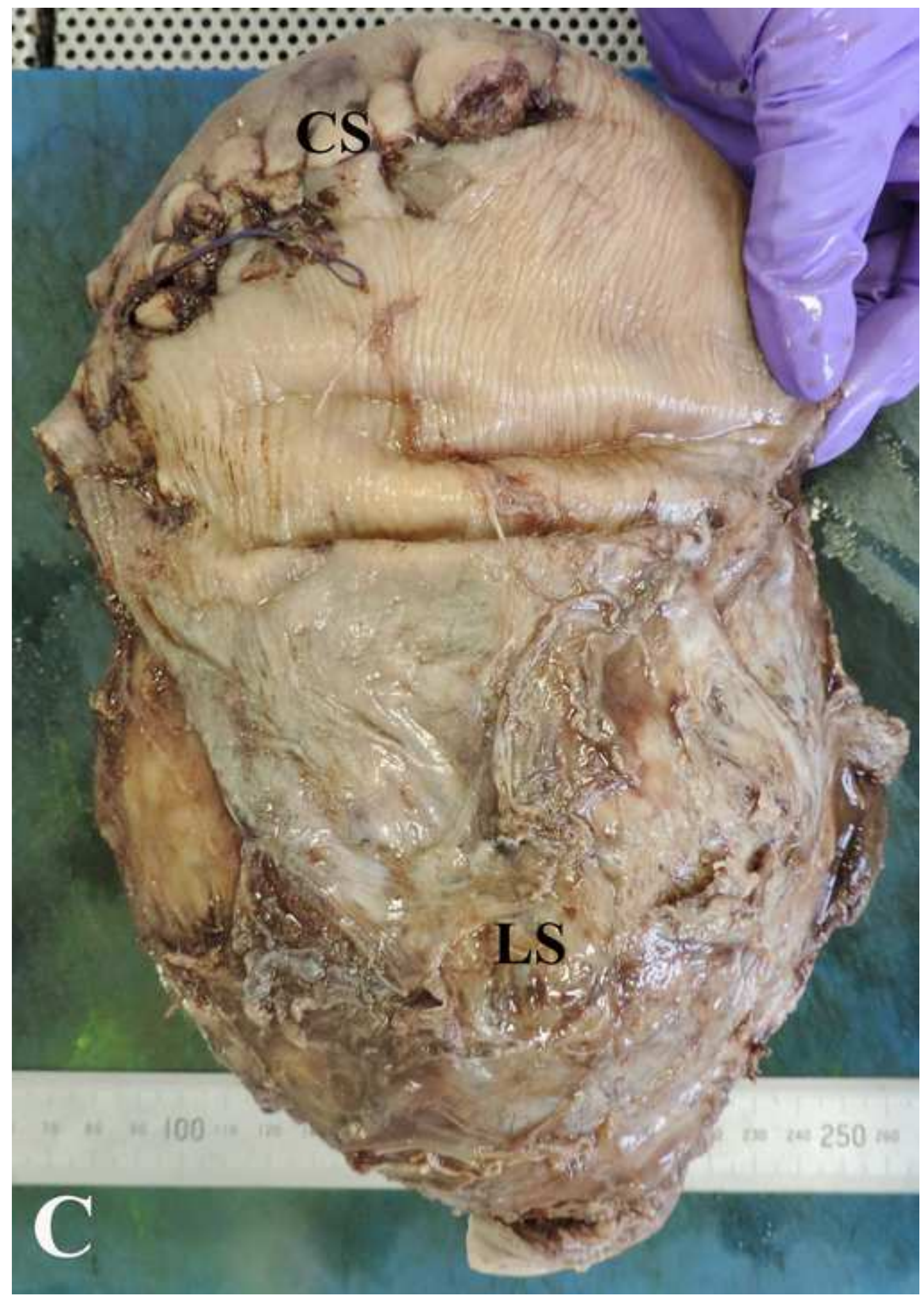


Click here to download high resolution image

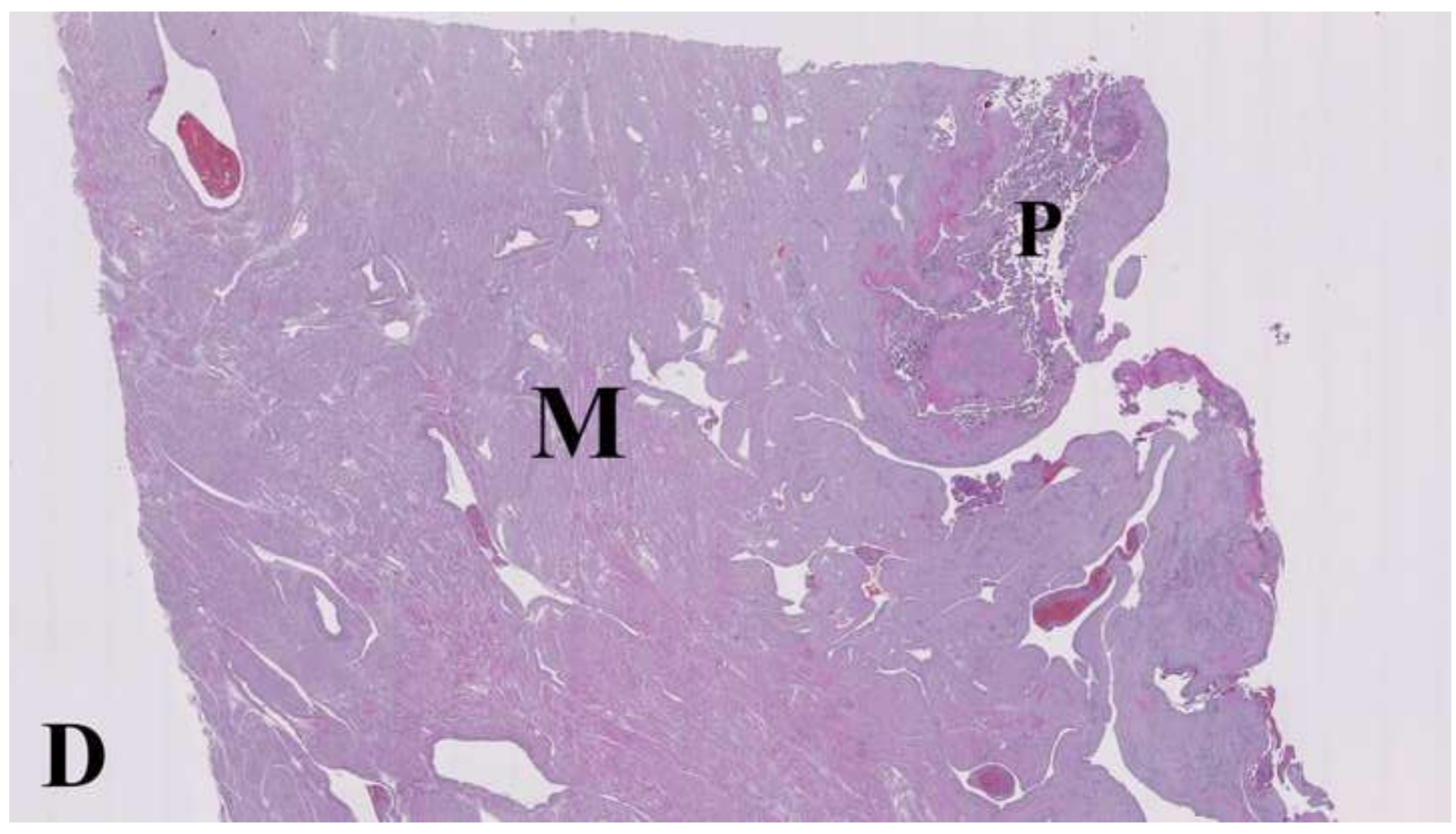


Click here to download high resolution image

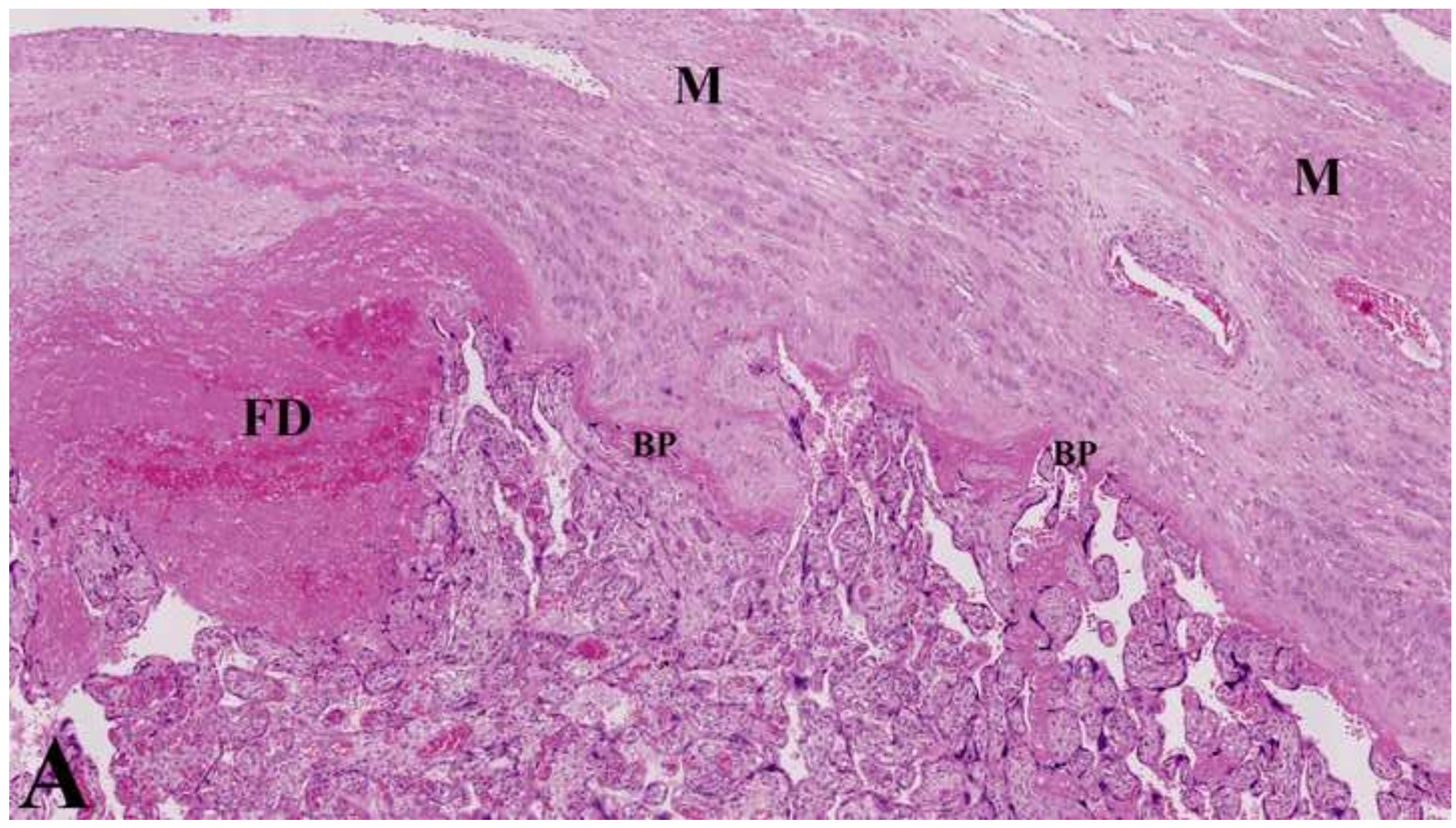


Figure 2B

Click here to download high resolution image

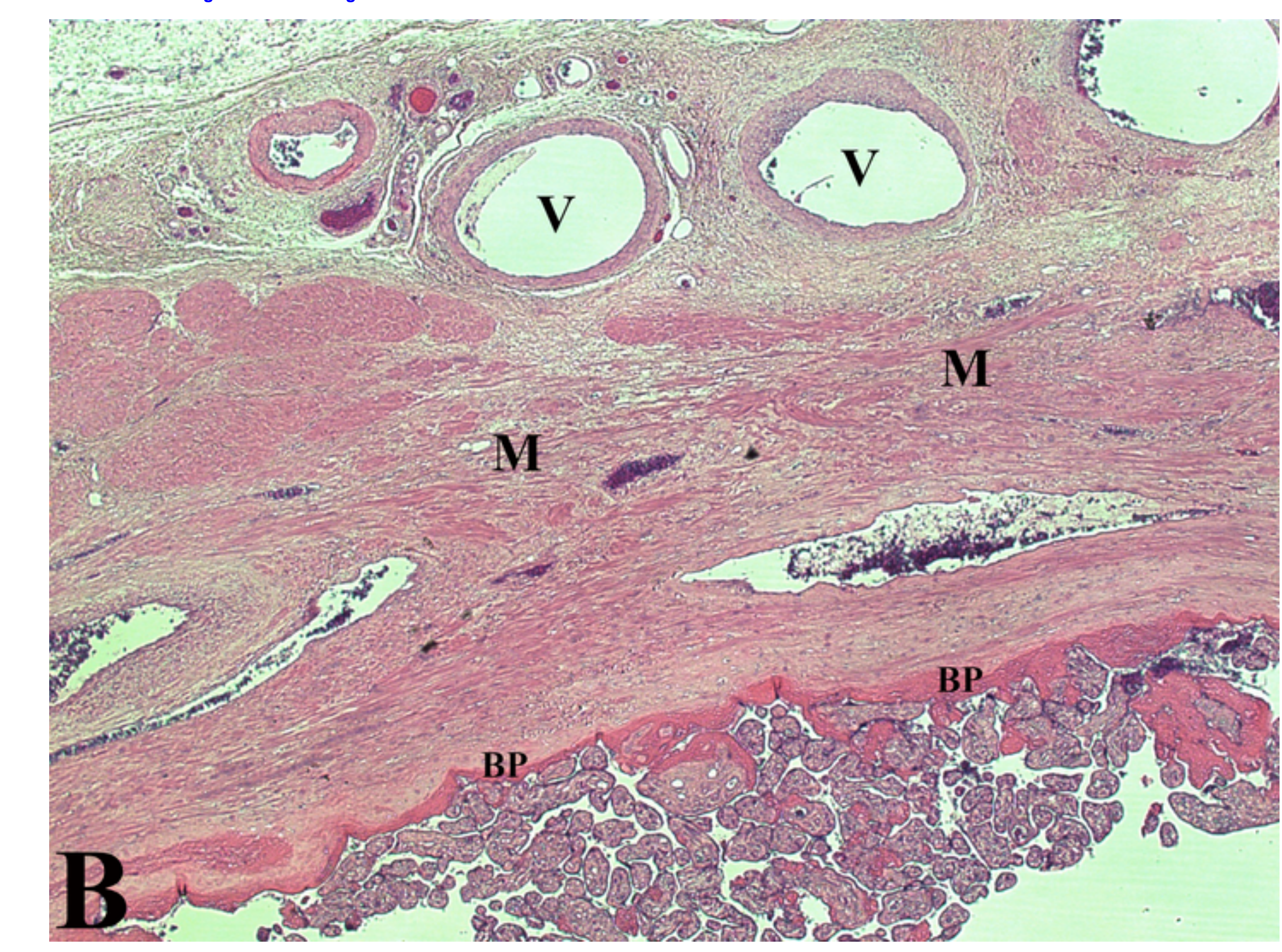




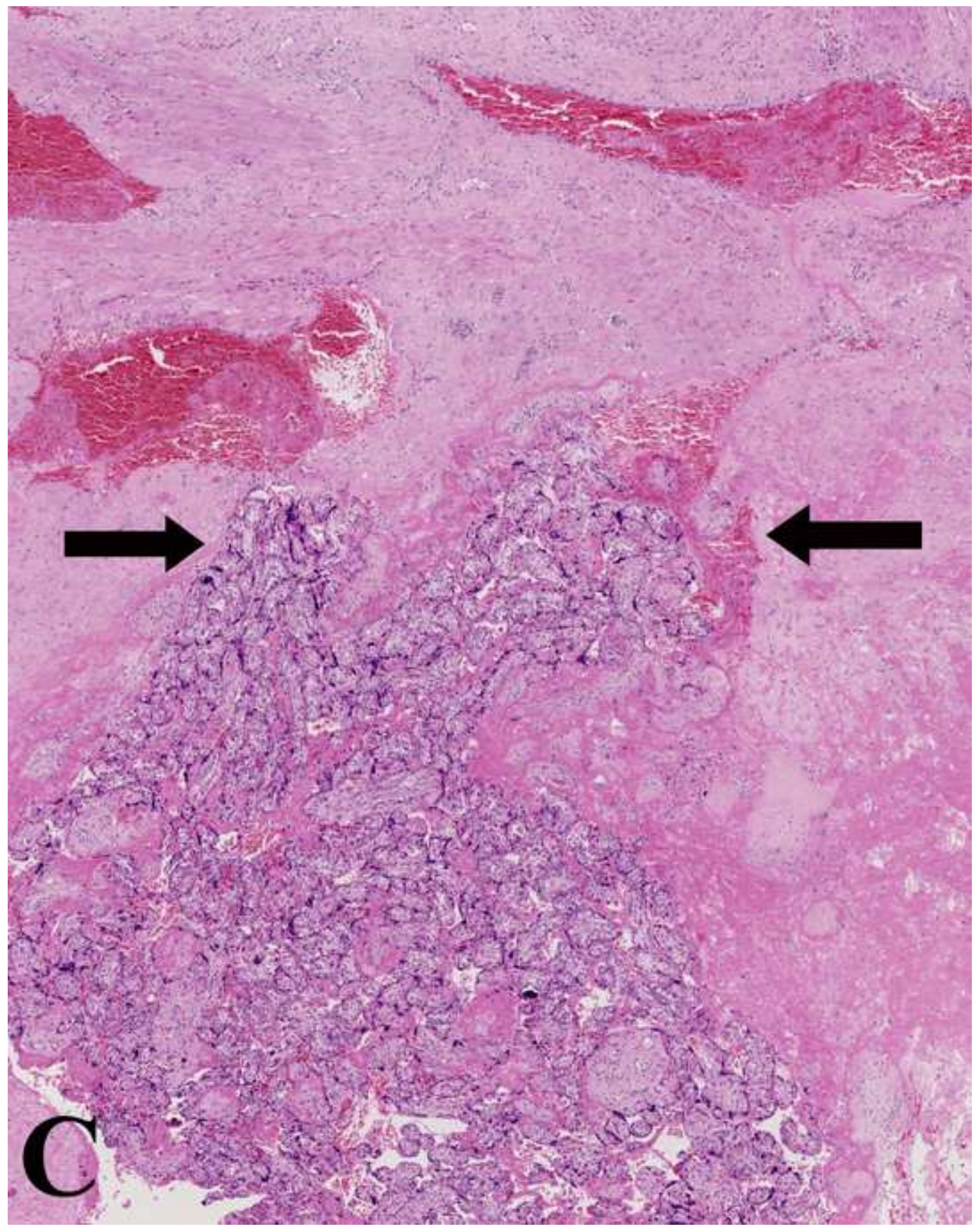




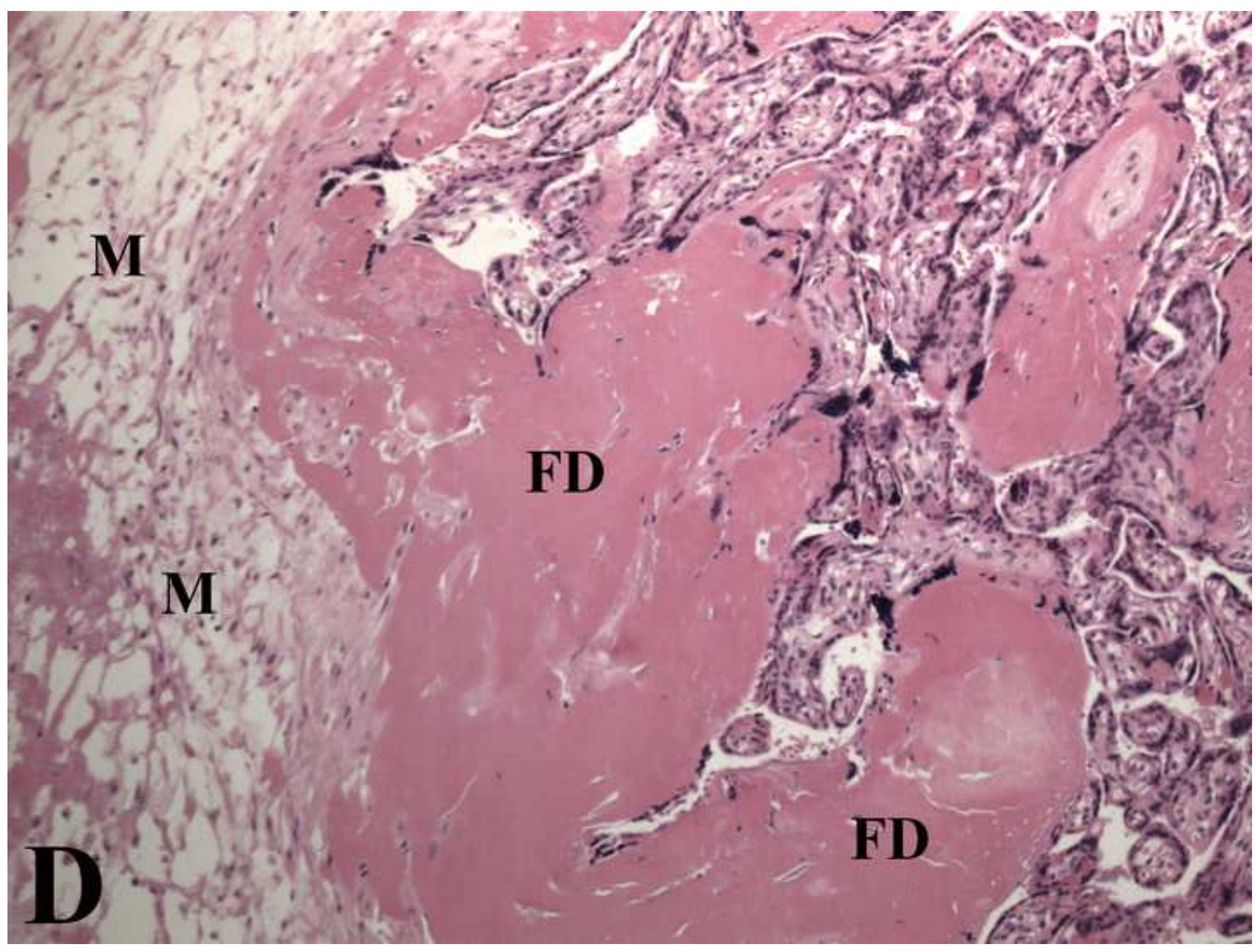

\section{Click here to download high resolution image}


Click here to download high resolution image

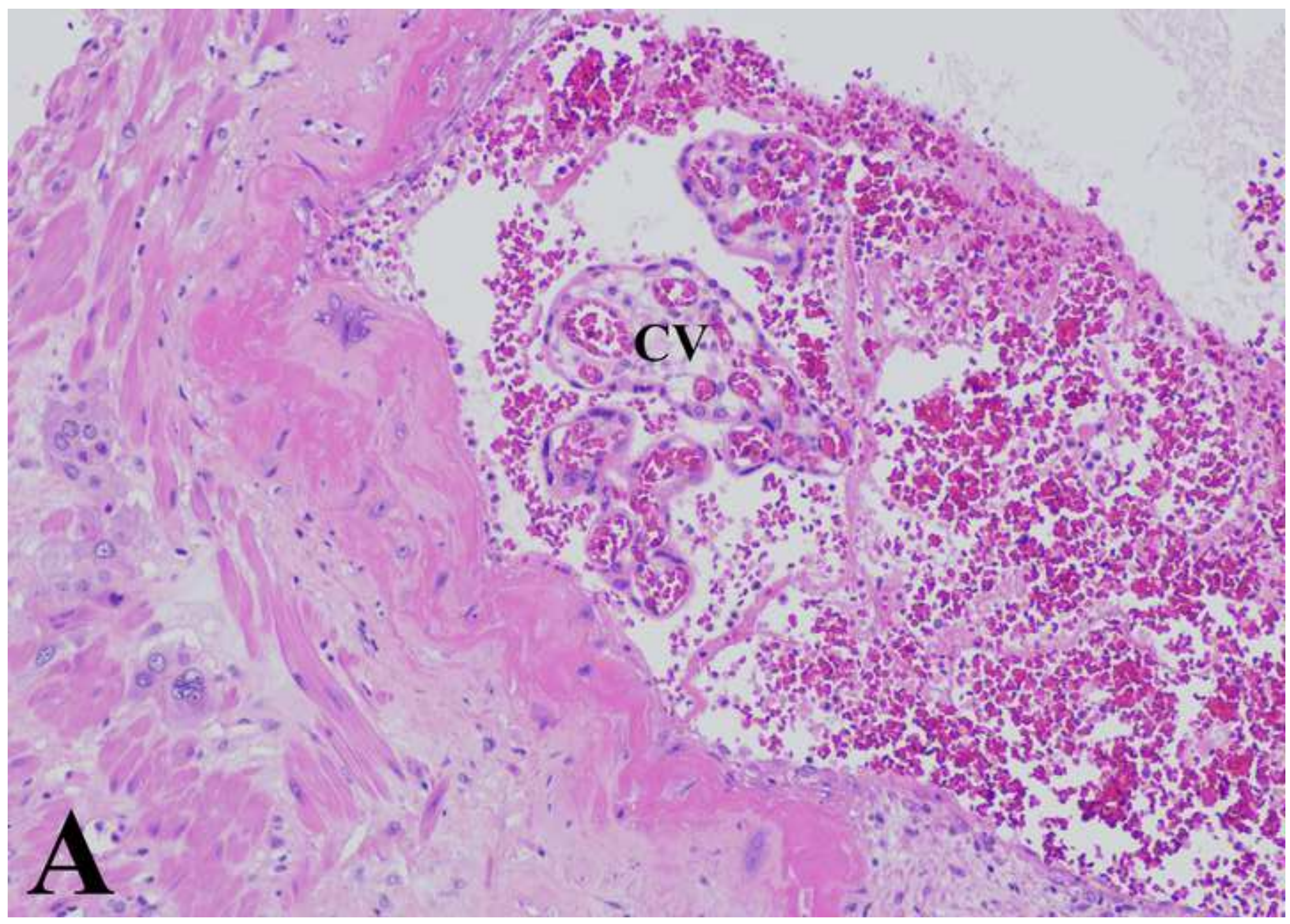




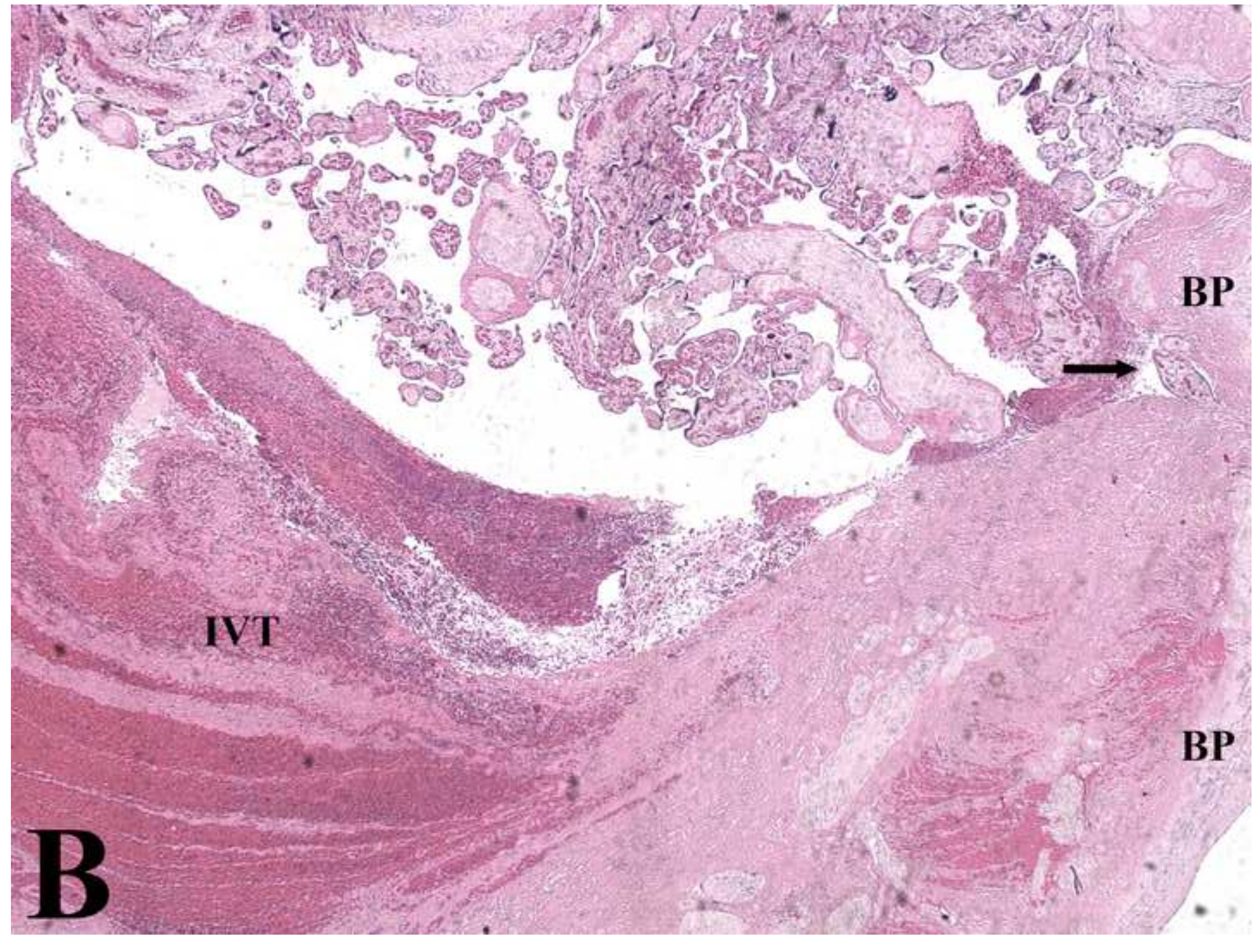

\section{Click here to download high resolution image}

A. Sn to 


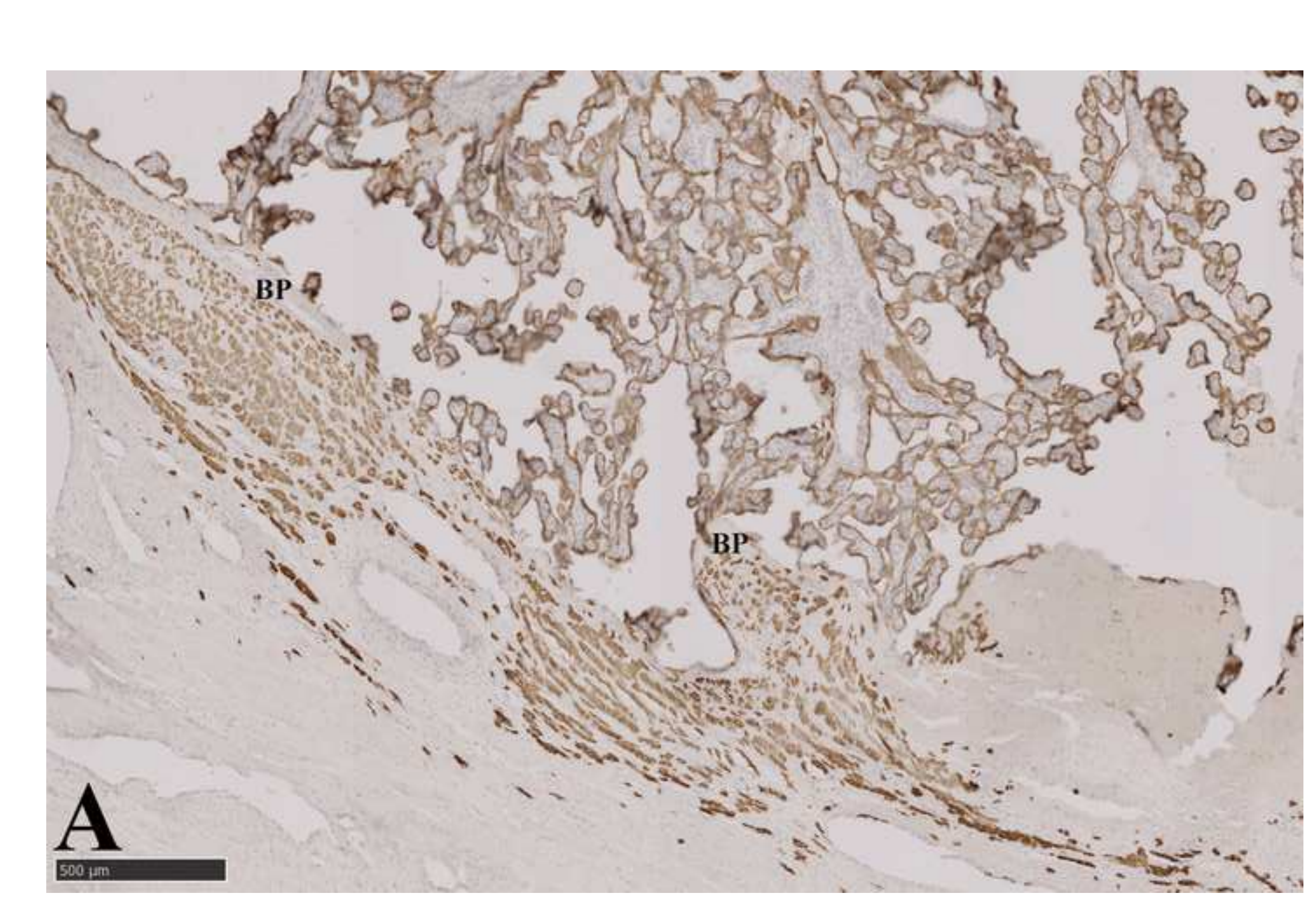




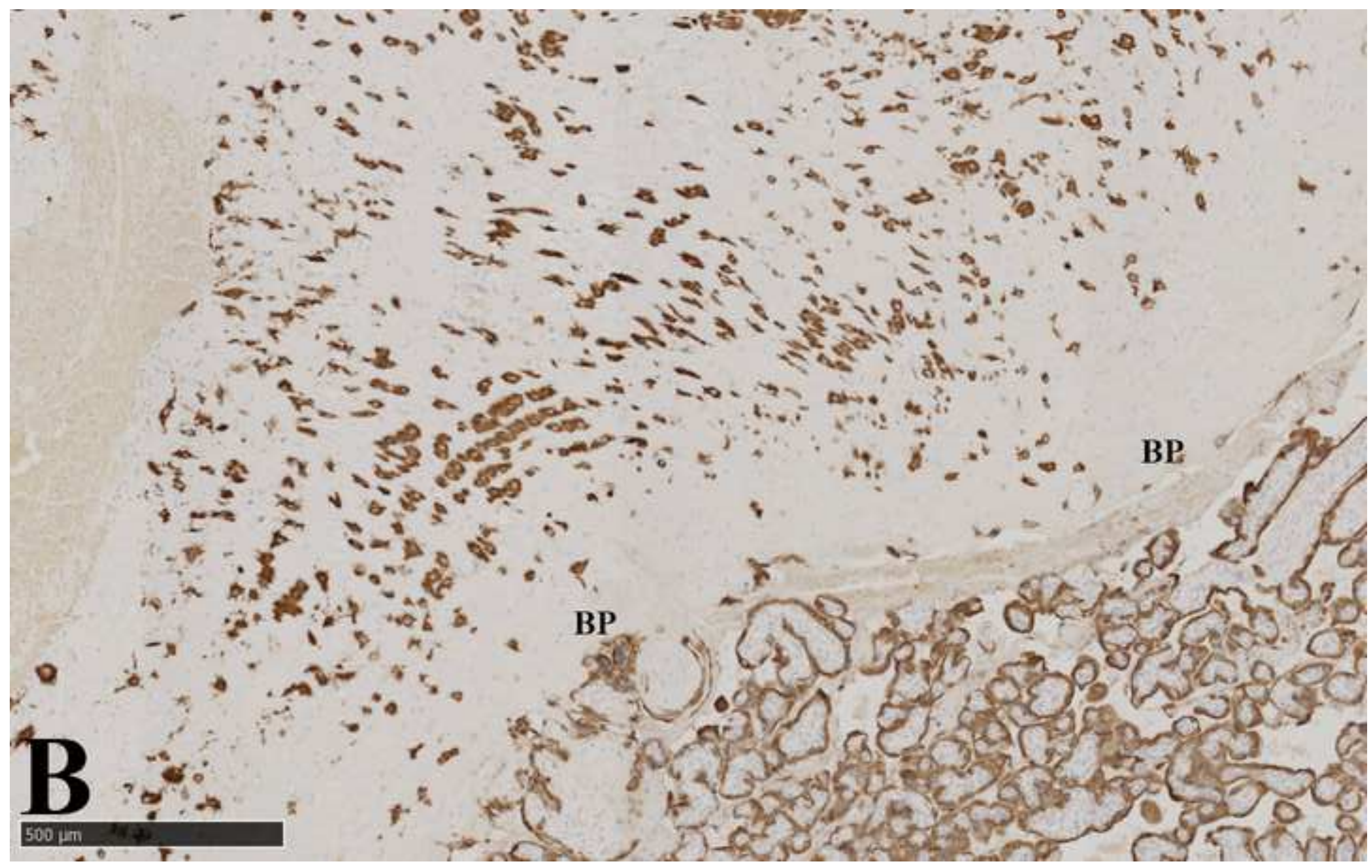

$4{ }^{6} \mathbf{r}$.

Be

(1)

\section{(1)}

(1)

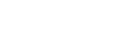

